ARTICLE

\title{
Synthesis of high-titer alka(e)nes in Yarrowia lipolytica is enabled by a discovered mechanism
}

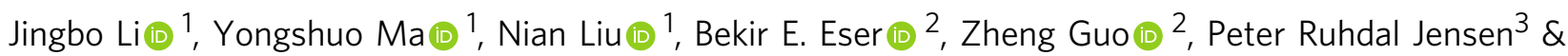
Gregory Stephanopoulos (i) ${ }^{1 \times}$

Alka(e)nes are ideal fuel components for aviation, long-distance transport, and shipping. They are typically derived from fossil fuels and accounting for $24 \%$ of difficult-to-eliminate greenhouse gas emissions. The synthesis of alka(e)nes in Yarrowia lipolytica from $\mathrm{CO}_{2}$ neutral feedstocks represents an attractive alternative. Here we report that the high-titer synthesis of alka(e)nes in Yarrowia lipolytica harboring a fatty acid photodecarboxylase (CVFAP) is enabled by a discovered pathway. We find that acyl-CoAs, rather than free fatty acids (FFAs), are the preferred substrate for CVFAP. This finding allows us to debottleneck the pathway and optimize fermentation conditions so that we are able to redirect $89 \%$ of acyl-CoAs from the synthesis of neutral lipids to alka(e)nes and reach titers of $1.47 \mathrm{~g} / \mathrm{L}$ from glucose. Two other $\mathrm{CO}_{2}$-derived substrates, wheat straw and acetate, are also demonstrated to be effective in producing alka(e)nes. Overall, our technology could advance net-zero emissions by providing $\mathrm{CO}_{2}$-neutral and energy-dense liquid biofuels.

\footnotetext{
${ }^{1}$ Department of Chemical Engineering, Massachusetts Institute of Technology, Cambridge, MA 02142, USA. ${ }^{2}$ Department of Engineering, Aarhus University, Gustav Wieds Vej 10, Aarhus 8000, Denmark. ${ }^{3}$ National Food Institute, Technical University of Denmark, Kongens Lyngby 2800, Denmark.

$凶_{\text {email: gregstep@mit.edu }}$
} 
ncreasing greenhouse gas (GHG) emissions is the leading cause of climate change ${ }^{1}$ and efforts to minimize the use of fossil fuels have mainly focused on deploying alternative energy sources such as bioethanol and electrical power. However, due to their low energy densities, both of these approaches struggle to transform the aviation, trucking, and shipping industries, making long-distance transport particularly difficult to decarbonize $^{2}$. It has been reported that air transportation and other modes of heavy-duty transport emitted 830 and $1060 \mathrm{Mt}$ of $\mathrm{CO}_{2}$ in 2014, respectively, accounting for $24 \%$ of difficult-toeliminate GHG emissions, and these numbers are projected to increase in the foreseeable future ${ }^{2}$. Although up to $0.8 \%$ reduction of overall emissions in the aviation industry could be achieved by blending conventional fuels with $1 \%$ of bio-jet fuels ${ }^{3}$, bio-jet fuels such as bio-kerosene are currently derived from vegetable oils, intensifying concerns over competition with food supply ${ }^{4}$. Therefore, the production of alka(e)nes with chain lengths in the C5-C23 range from sugar, biomass, or $\mathrm{CO}_{2}$-derived feedstocks could have better potential in disrupting the current state of transportation, leading to superior mitigation of $\mathrm{CO}_{2}$ emissions. Generally, renewable alka(e)nes can be synthesized either chemically or biologically. The former typically involves sequential hydrolysis and dehydration of biomass followed by C-C bond formation and hydrodeoxygenation, which require elevated temperatures and pressures, as well as different types of catalysts, complicating the overall process ${ }^{5}$. By contrast, bioconversion approaches offer high carbon- and energy-conversion efficiencies in single-unit operations, yielding an overall simplified process ${ }^{6}$.

Three classes of hydrocarbons can be biologically synthesized: those derived from fatty acids, isoprenoids, and polyketides, respectively ${ }^{4}$. For fatty acid-derived hydrocarbons, two major metabolic pathways have been identified, the two-step cyanobacterial pathway involving acyl-ACP reduction and aldehyde decarbonylation ${ }^{7}$, and the one-step decarboxylation reaction from free fatty acids (FFAs) ${ }^{8-11}$. In prior studies, both pathways were harnessed in $E$. coli to synthesize alka(e)nes with varying chain lengths, with reported titers ranging from 1.3 to $1310 \mathrm{mg} / \mathrm{L}$ and corresponding yields ranging from 0.43 to $11 \mathrm{mg} / \mathrm{g}$ glucose (Supplementary Table 1). Another model microorganism, Saccharomyces cerevisiae, was engineered to produce alka(e)nes as well, albeit achieving relatively lower titers and yields compared to those obtained with E. coli (Supplementary Table 1). Yarrowia lipolytica, a widely recognized model oleaginous organism ${ }^{12}$ due to its high lipid synthesis capacity, was also used and shown to be able to produce $23 \mathrm{mg} / \mathrm{L}$ alka(e)nes through the cyanobacterial pathway ${ }^{13}$. Very recently, a fatty acid photodecarboxylase from Chlorella variabilis NC64A (CvFAP) was discovered to decarboxylate FFAs with preference to palmitic and heptadecanoic acids over lauric, myristic, stearic, oleic, and linoleic acids ${ }^{11}$. It was then expressed in an FFA-overproducing $Y$. lipolytica strain $^{14}$. Although the production of $10.87 \mathrm{mg} / \mathrm{L}$ and $58.7 \mathrm{mg} / \mathrm{L}$ alka(e)nes were achieved in the presence of light with batch and fed-batch fermentation modes, respectively, no information regarding the responsible metabolic pathways was detailed ${ }^{14}$. Furthermore, it is unknown as to why adding crude cell lysate to the purified enzyme accelerates palmitic acid to pentadecane conversion in vitro ${ }^{15}$. In order to study how $C v$ FAP functions and uncover its relations to alka(e)ne synthesis in $Y$. lipolytica, an oleaginous yeast that produces approximately $30 \mathrm{mg} / \mathrm{L}$ FFAs before metabolic rewiring ${ }^{16}$, in this work, we set out to harness the photodecarboxylation pathway as well as its supporting pathways in Y. lipolytica. By identifying acyl-CoAs as one of the substrates for $C v \mathrm{FAP}$, we are able to significantly improve the decarboxylation flux such that $89 \%$ of acyl-CoAs are directed away from the synthesis of neutral lipids and to alka(e)nes under optimal conditions. Additionally, our strain is compatible with two $\mathrm{CO}_{2}$ fixation schemes, one based on biomass hydrolysates and the other on $\mathrm{CO}_{2}$-derived acetic acid, both of which can be fed to Y. lipolytica directly for alka(e)ne production.

\section{Results}

Engineering and rewiring pathways for alka(e)ne synthesis in Y. lipolytica. Expression of the codon-optimized CvFAP in $Y$. lipolytica polf resulted in YLjbl-2 which produced $15.3 \mathrm{mg} / \mathrm{L}$ alka (e)nes. The constituents were verified to be heptadecane (C17:0), 8-heptadecene (C17:1), 6,9-heptadecadiene (C17:2), and pentadecane (C15:0) by comparing their mass spectra (Supplementary Fig. 1) to a NIST library as well as a previous report ${ }^{17}$.

Since FFAs were believed to be the substrate of $C \nu \mathrm{FAP}$ in in vitro studies $^{11,15}$, harnessing FFA-producing pathways and blocking FFA-consuming pathways in YLjbl-2 were expected to improve alka(e)ne synthesis. To investigate this hypothesis, we expressed enzymes for FFAs synthesis including the bifunctional acyl-ACP/ acyl-CoA thioesterase $(E c$ TesA') from E. coli and the acyl-ACP thioesterase from Umbellularia californica $(U c \mathrm{ACPT})^{13,18}$ in strain YLjbl-2. However, the thioesterase expression depressed alka(e)ne synthesis, though FFA titers were enhanced (Fig. 1b). We then disrupted FAA1, the cytosolic enzyme responsible for converting FFAs to acyl-CoAs ${ }^{19}$, in strains overexpressed thioesterases. Compared with strains overexpressed thioesterase, FAA1 knockout improved the alka(e)ne titer and FFA titer in both cases. However, compared with YLjbl-2, neither the thioesterase expression nor the FAA1 knockout improved alka (e)ne synthesis, though FFA titers were enhanced (Fig. 1b). Together with FAA1 knockout, we also expressed the FAS$E c T_{e s}^{\prime}$ and FAS-UcACPT fusion proteins, which were found to be effective for FFA production in Y. lipolytica ${ }^{13}$, but the resulting strains produced less alka(e)nes as well (Supplementary Fig. 2). Targeting both thioesterases to the endoplasmic reticulum where triglycerides are synthesized increased FFA formation but did not enhance alka(e)ne production either (Supplementary Fig. 2). Expression of lipases ${ }^{20}$ in YLjbl-2 was also carried out, but no increases in alka(e)ne titers were observed despite the improved FFA production (Supplementary Fig. 2). We also noticed that the expression of the $C v \mathrm{FAP}$ in an FFA-overproducing strain that was able to produce around $2 \mathrm{~g} / \mathrm{L}$ intracellular and $2.7 \mathrm{~g} / \mathrm{L}$ extracellular FFAs ${ }^{20}$ gave rise to $10.87 \mathrm{mg} / \mathrm{L}$ alka(e)nes from $50 \mathrm{~g} /$ $\mathrm{L}$ glucose ${ }^{14}$, which is lower than the titer produced by YLjbl-2 from $20 \mathrm{~g} / \mathrm{L}$ glucose (Fig. 1b). These results suggest that the overproduction of FFAs is not a prerequisite for obtaining more alka(e)nes. Together with the observation that the purified $C \nu$ FAP enzyme showed 6-12 times lower activity than the crude cell lysate in an earlier report ${ }^{15}$, we speculate that there may be an unknown mechanism regarding the CvFAP enzyme when converting FFAs to alka(e)nes.

A proposed metabolic pathway for alka(e)ne synthesis in $Y$. lipolytica. In order to explore the mechanism, we designed experiments where labeled palmitic acid- $\mathrm{d}_{31}$ was metabolized by YLjbl-2 and YLjbl-2- $\triangle F A A 1$. Both strains produced pentadecane$\mathrm{d}_{31}$ (C15:0) directly from palmitic acid- $\mathrm{d}_{31}$ through decarboxylation (Fig. 2a, b, Supplementary Fig. 3a). We also detected stearic acid methyl ester- $\mathrm{d}_{31}$ in methylated samples from YLjbl-2 cells but not from YLjbl-2- $\triangle F A A 1$ cells (Supplementary Fig. 3b). Since the CoA form is required for acyl chain elongation from $\mathrm{C} 16$ to $\mathrm{C} 18$, this confirms the existence of stearoyl-CoA- $\mathrm{d}_{31}$ in YLjbl-2 cells.

Interestingly, a formed compound with the retention time of 8.36 min was observed by supplementing YLjbl-2 with palmitic acid- $d_{31}$ (Fig. 2b). We further identified the peak through GC-MS and confirmed it to be heptadecane- $\mathrm{d}_{31}$ (C17:0) according to its 


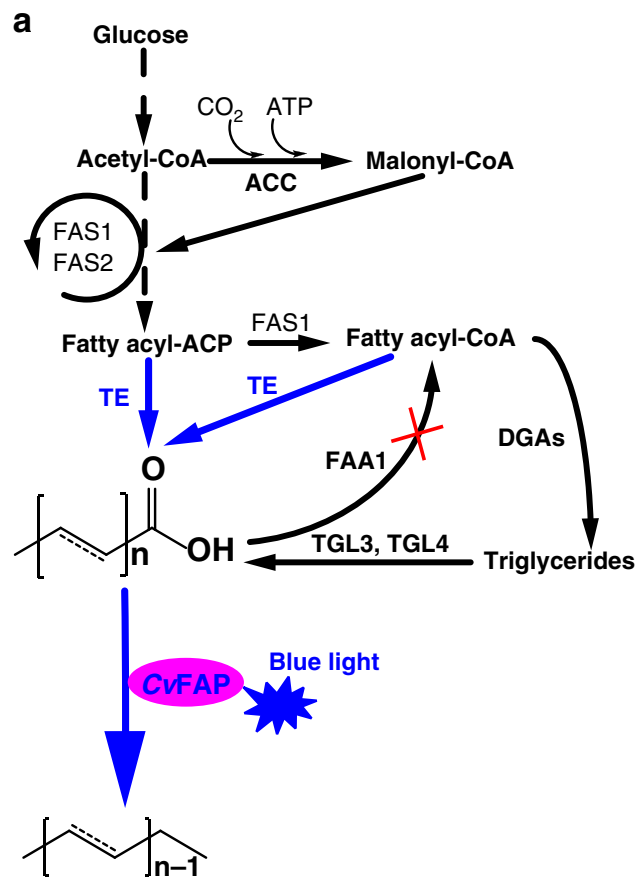

b

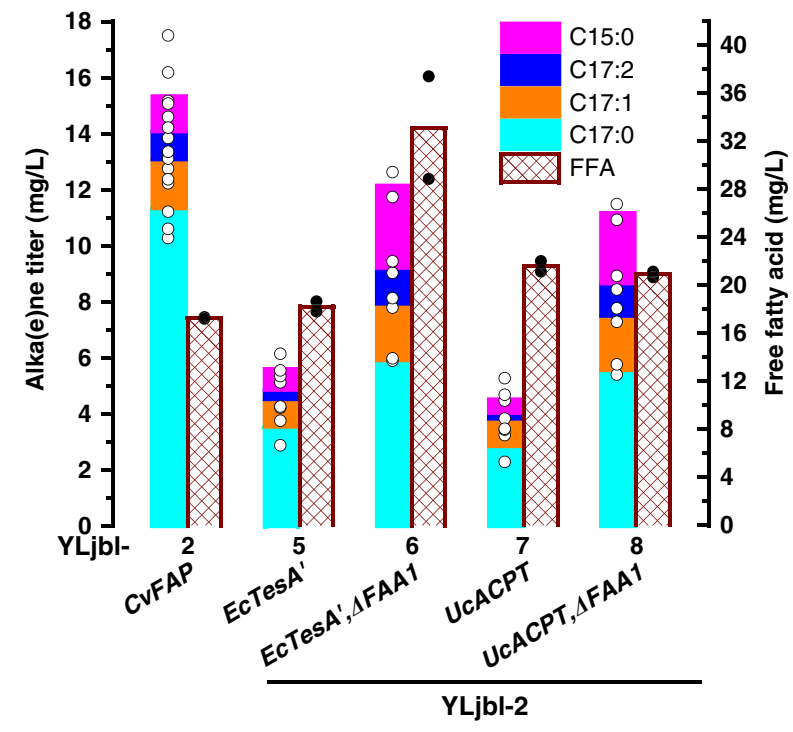

Alkanes/alkenes

Fig. 1 The effect of expression of thioesterases on alka(e)ne production. a Metabolic pathways for alka(e)ne production employing the fatty acid photodecarboxylase according to previous literature that free fatty acids are the precursors of alka(e)nes (the revised pathway is shown in Fig. 2). Black arrows represent native pathways while blue arrows indicate heterologous pathways. TE, acyl-CoA/acyl-ACP thioesterases; TGL3 and TGL4, intracellular lipases from Y. lipolytica; FAA1, acyl-CoA synthase, ACC, acetyl-CoA carboxylase, FAS1 and FAS2, fatty acid synthase 1 and 2, DGAs, diacylglycerol transferases. $\mathbf{b} A \mathrm{Alka}(\mathrm{e})$ ne production is observed by expressing photodecarboxylase in a wild-type strain. Expression of thioesterases (EcTes $\mathrm{A}^{\prime}$ and UCACPT) and knockout of FAA1 did not enhance alka(e)ne production but decreased, although free fatty acid titers were increased. Fermentations were performed in $50 \mathrm{~mL}$ conical shake flasks with a working volume of $13 \mathrm{~mL}$ and an initial $\mathrm{OD}_{600}$ of 0.1 for 3 days in the dark followed by 3 days in blue light generated by light source 1 (Supplementary Fig. 16). Fermentation medium was composed of $20 \mathrm{~g} / \mathrm{L}$ glucose, $6.9 \mathrm{~g} / \mathrm{L}$ yeast nitrogen base (without amino acids), and $1 \mathrm{~g} / \mathrm{L}$ yeast extract. Data represent mean value, $n=2$ biologically independent samples. Source data underlying Fig. $1 \mathrm{~b}$ are provided as a Source Data file.

molecular weight and MS fragments (Supplementary Fig. 3c). Since this product comes from the decarboxylation of a C18 acyl chain and no stearic acid- $\mathrm{d}_{31}$ was detected (Supplementary Fig. 3d), we hypothesized that stearoyl-CoA- $\mathrm{d}_{31}$ could be the precursor for synthesizing heptadecane- $\mathrm{d}_{31}$ by $C v \mathrm{FAP}$. In order to provide evidence for this hypothesis, we purified the enzyme and subjected it to stearoyl-CoA lithium salt as the substrate and verified the formation of heptadecane- $\mathrm{d}_{31}(\mathrm{C} 17: 0)$ (Fig. 2c, Supplementary Fig. 3e). Based on these results, we proposed the full pathway where pentadecane- $\mathrm{d}_{31}(\mathrm{C} 15: 0)$ can be synthesized from both palmitic acid- $\mathrm{d}_{31}$ and palmityl-CoA- $\mathrm{d}_{31}$, while heptadecane- $\mathrm{d}_{31}(\mathrm{C} 17: 0)$ can only be derived from stearoylCoA- $\mathrm{d}_{31}$ in $Y$. lipolytica as shown in Fig. 2d.

To determine whether FFA or acyl-CoA is the preferred substrate, we examined the titers of pentadecane- $\mathrm{d}_{31}$ and heptadecane- $\mathrm{d}_{31}$ over time in vivo and calculated their respective cumulative formation rates (Fig. 2e). Our measurements showed that the titer and the cumulative formation rate of heptadecane- $\mathrm{d}_{31}$ (C17:0) were 2-fold and 3.5-fold higher than those of pentadecane- $\mathrm{d}_{31}(\mathrm{C} 15: 0)$, respectively. Together with our proposed pathway in Fig. 2 d, it seems that $C \nu$ FAP prefers acyl-CoA over FFA. In order to confirm this, we studied the enzyme kinetics of $C v$ FAP using stearoyl-CoA lithium salt and lithium stearate as substrates. The enzyme displayed Michaelis-Menten kinetics with a $K_{\mathrm{m}}$ constant of 75.30 or $108.31 \mu \mathrm{M}$, a $V_{\max }$ of 3.22 or $2.83 \mu \mathrm{M} / \mathrm{h}$, and a $k_{\text {cat }}$ of 1114.62 and $979.62 \mathrm{~s}^{-1}$ when the substrate was stearoyl-CoA or stearic acid, respectively (Fig. 2 f, g). Thus, specificity constant $\left(k_{\text {cat }} /\right.$ $K_{\mathrm{m}}$ ) for stearoyl-CoA is about 1.6-fold higher compared to specificity constant for stearic acid (14.8 vs 9.0), providing further evidence that acyl-CoA is the preferred substrate for $C v \mathrm{FAP}$.

We further proposed a catalytic mechanism as illustrated in Supplementary Note 1. In the first step, acyl-CoA binds to the active site and gets hydrolyzed with the help of the polar residues and water/hydroxide around thioester bond (Supplementary Fig. 4). In the next step, CoA dissociates from the active site, whereas FFA remains bound inside the hydrophobic substrate tunnel. This is followed by binding of FAD into the region of the active site that has been abandoned by CoA. Thereafter the lightdependent mechanism takes place through FAD-dependent radical chemistry leading to decarboxylation of FFA into alkane, as described earlier ${ }^{11}$. Docking of palmitoyl-CoA into the $C v$ FAP structure indicates the presence of multiple polar side chains around thioester bond, which can possibly support a hydrolysis mechanism. Given the diversity of active site motifs present in a wide variety of thioesterases ${ }^{21}$, further mechanistic and structural studies will be required to reveal the exact nature of the hydrolysis step.

Our proposed mechanism, where a larger substrate is hydrolyzed before it goes through complex chemistry in a single enzyme active site, is not uncommon in literature ${ }^{22}$. CalE7, a bacterial thioesterase that plays a role in enediyne biosynthesis, first hydrolyzes the thioester bond of its ACP- 
a

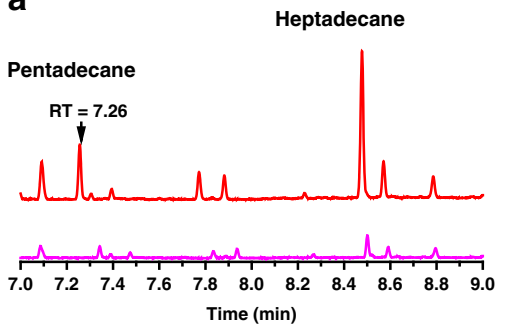

d

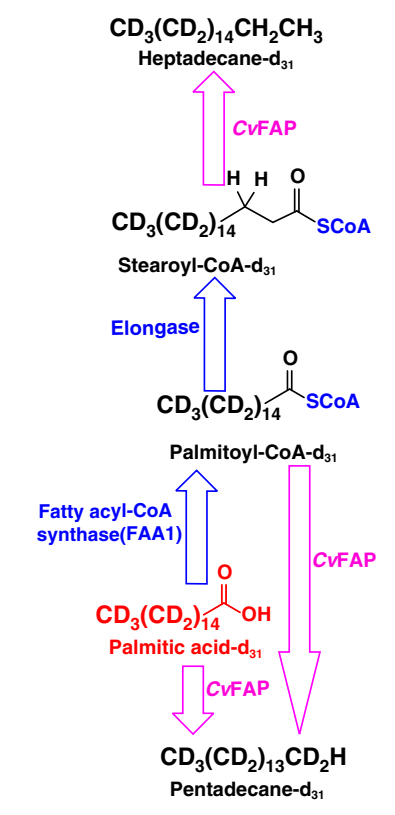

b

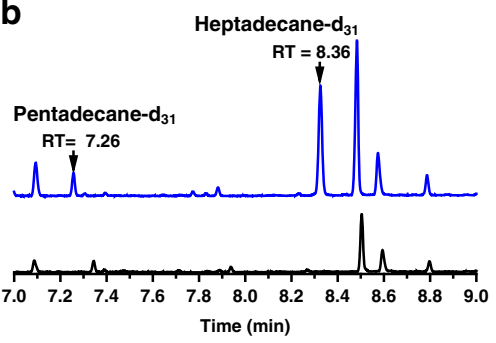

e

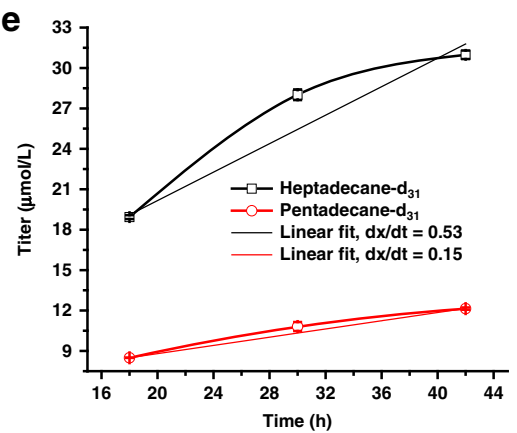

g

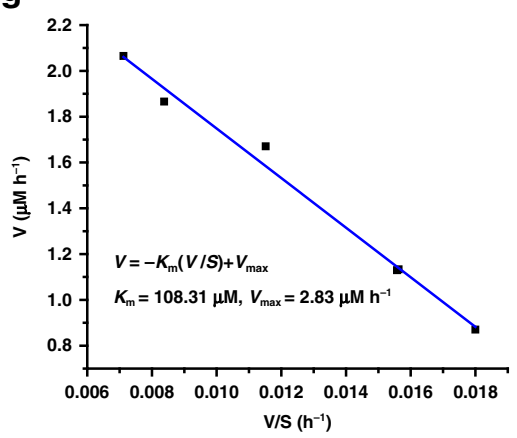

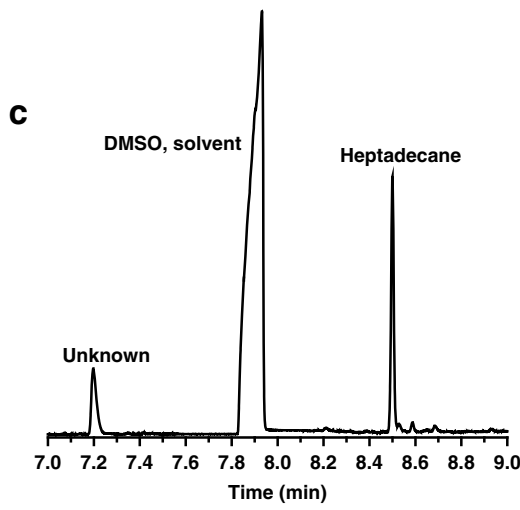

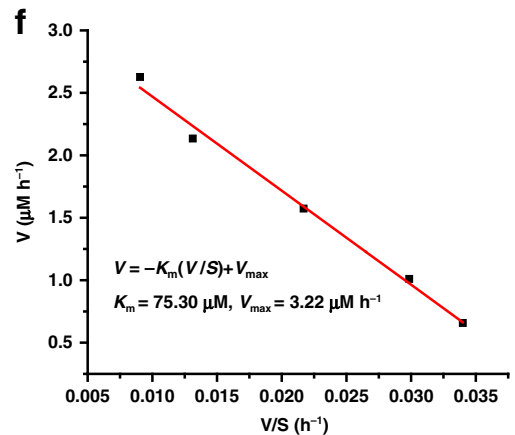

h

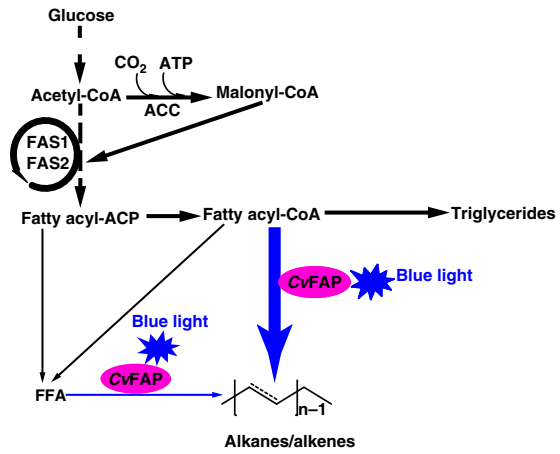

Fig. 2 A proposed metabolic pathway for alka(e)ne synthesis in Y. lipolytica. a, b GC chromatograms from strain YLjbl-2- $\triangle F A A 1$ (red) and strain YLjbl-2 (blue) cultivated with exogenous palmitic acid- $d_{31}$ supplementation, respectively. Magenta and black lines represent the same cultivation without palmitic acid- $d_{31}$ addition. Two compounds with retention time of 7.26 and 8.36 min were found and identified to be pentadecane- $d_{31}$ and heptadecane- $d_{31}$, respectively. c GC chromatogram of products from the in vitro photocatalytic reaction using stearoyl-CoA lithium salt ( $\geq 90 \%$, Sigma-Aldrich) as the substrate and purified CVFAP as the catalyst, showing that heptadecane could be synthesized from stearoyl-CoA. $\mathbf{d}$ Proposed mechanism for pentadecane$d_{31}$ and heptadecane- $d_{31}$ formation from exogenous palmitic acid- $d_{31}$. Blue arrows indicate endogenous pathways while magenta arrows represent photodecarboxylation. e In vivo pentadecane- $d_{31}$ and heptadecane- $d_{31}$ formation rates resulting from the strain YLjbl-2 indicated that fatty acyl-CoA might be the preferred substrate for the photodecarboxylase. $\mathbf{f}, \mathbf{g}$ Kinetic analyses of purified CVFAP at fixed concentrations for the determination of $K_{m}$ and $V_{\text {max }}$ when stearoyl-CoA lithium salt and lithium stearate $\left(\geq 98.0 \%, \mathrm{TCl}\right.$ America) were used as substrates, respectively. Values of $K_{m}$ and $V_{\text {max }}$ were determined through Eadie-Hofstee plots. The results suggested that fatty acyl-CoAs were the preferred substrate for CVFAP over free fatty acids. h: A proposed pathway for alka(e)ne production catalyzed by CVFAP. Blue arrow represents photodecarboxylation and bold blue arrow indicates higher metabolic flux. ACC, acetyl-CoA carboxylase, FAS1 and FAS2, fatty acid synthase 1 and 2. Data represent mean value, $n=2$ biologically independent samples. Source data underlying Fig. 2e-g are provided as a Source Data file.

tethered acyl substrate before catalyzing decarboxylation of the resulting $\beta$-ketocarboxylic acid intermediate ${ }^{22}$. An active site arginine acting as an oxyanion hole and water or a hydroxide acting as nucleophile have been proposed to catalyze the thioester hydrolysis step. Another literature example is thiazole biosynthetic enzyme, Thi4, present in yeast and archaea. This enzyme firstly hydrolyzes its substrate NAD into nicotinamide and ADP-ribose. Afterwards, ADP-ribose goes through a complex transformation that involves binding of iron and other substrates into the active site to carry out multiple steps including iron-dependent sulfur transfer ${ }^{23}$. Analogous to $C v \mathrm{FAP}$, Thi4 can also take ADP-ribose as a substrate instead of NAD (acyl-CoA vs. FFA).

These findings support the proposed metabolic pathway for alka(e)ne synthesis when the photodecarboxylase is used (Fig. 2h). It indicates that acyl-CoA is the primary precursor for producing alk(e)nes in Y. lipolytica due to its abundant availability and and its higher specificity constant towards $C v$ FAP. Thus, improvements to the acyl-CoA pool or $C v$ FAP efficiency may further increase alka(e)ne titers. 
Debottlenecking alka(e)ne synthesis by increasing the copy number of CvFAP. Having established that acyl-CoA is the preferred substrate of $C v \mathrm{FAP}$, we sought to increase its availability by knocking out $D G A 1$ and $D G A 2$, which encode enzymes that are responsible for sequestering acyl-CoAs in triglycerides. However, both single and double deletions resulted in lower alka (e)ne and lipid titers relative to the parental strains (Supplementary Fig. 5a). It was shown in a previous study ${ }^{24}$ that acyl$\mathrm{CoA}$ intermediates function as feedback inhibitors to fatty acid synthesis, and suppressed fatty acid synthesis further resulted in growth defects ${ }^{25}$. Deletion of DGA1, DGA2, or both decreased the overall pool of acyl-CoA, which led to lower availability of precursors for alka(e)ne synthesis. Additionally, we combined $\triangle D G A 1$ and/or $\triangle D G A 2$ with CvFAP overexpression via introducing the linearized CvFAP plasmid once more. This combination of gene modulations produced 1.5 to 3 -fold more alka(e) nes than the corresponding parental strains (Supplementary Fig. 5b). Lipid titer, on the contrary, decreased slightly, possibly due to the diversion of acyl-CoA away from triglycerides to alka (e)nes. Therefore, under the same acyl-CoA flux, higher $C \nu$ FAP activity drove more acyl-CoA to alka(e)nes, which also proved our finding that acyl-CoA was one of the precursors for $\mathrm{C} v \mathrm{FAP}$. Overall, these results support the effectiveness of increasing decarboxylation rate by increasing the CVFAP copy number.

We next sought to engineer the strain further by knocking out enzymes that are related to alka(e)ne assimilation (Fig. 3a). Though the active FAA1 was expected to be beneficial for alka(e) ne production according to the finding that acyl-CoA was the preferred substrate for $C v \mathrm{FAP}$, deletion of FAA1 did not decrease alka(e)ne titers (Fig. 3b). It may be because that no pathways for FFA formation were incorporated in these strains such that the deletion of FAA1 did not decrease the acyl-CoA pool. Surprisingly, the alka(e)ne titers increased by the double deletion of FAA1 and ALK2 (Fig. 3b), which might be attributed to the synergistic effect between FAA1 and ALK2 based on the fact that FAA1 is relevant to the assimilation of n-alkanes ${ }^{19}$. Furthermore, we found that the total lipid titer of a strain overexpressing FAA1 was significantly higher than its parental strain with $\triangle F A A 1$ (Supplementary Fig. 6), whereas the alka(e)ne titer decreased by more than 3 -fold. This result indicates that $\triangle F A A 1$ plays a significant role in regulating the distribution of acyl-CoA to either alka(e)nes or triglycerides. Overall, $\triangle F A A 1$ mainly played two roles, acting with $\triangle A L K 2$ synergistically and regulating distribution of acyl-CoA between alka(e)nes and lipids.

Overexpression of CvFAP in strains with double deletions of FAA1 and ALK2 and triple deletions of FAA1, ALK1, and ALK2 enhanced alka(e)ne synthesis more profoundly (Fig. 3b). These results further corroborate the idea that the bottleneck for alka(e) ne production in Y. lipolytica is not the supply of precursors but rather the decarboxylation step. As such, we constructed plasmids carrying $2(\mathrm{CvFAPD})$ and $3(\mathrm{CvFAPT})$ copies of the $\mathrm{CvFAP}$ gene expression cassette (Supplementary Fig. 7a) and transformed them into different strains. Compared with the parental strain YLjbl-19, CvFAP, CvFAPD, and CvFAPT transformants showed 1.7, 2.4, and 2.4-fold higher alka(e)ne titers, respectively (Fig. 3c). Relative copy numbers of the CvFAP gene in genome and mRNA were quantified and showed a positive correlation with alka(e)ne titers (Fig. 3d, Supplementary Fig. 7b). In addition, pentadecene (C15:1) derived from palmitoleoyl-CoA was detected in these strains but not the parental strains (Fig. 3e), possibly attributed to the enhanced decarboxylation efficiency. Alka(e)ne production of $\mathrm{CvFAP}, \mathrm{CvFAPD}$, and CvFAPT transformants from YLjbl-20 was improved as well, but not as much as the transformants from YLjbl-19 (Fig. 3f). Overall, by increasing the CvFAP copy number coupled with the deletion of FAA1 and $A L K 2$, we were able to obtain a strain capable of producing $35 \mathrm{mg} / \mathrm{L}$ alka(e)nes from 20 $\mathrm{g} / \mathrm{L}$ glucose in a batch fermentation carried out in 12 -well plates.

Optimization of fermentation conditions for maximizing acylCoA to alka(e)ne flux. Strain YLjbl-26 was selected for further process optimization as it was most promising for alka(e)ne production. Our previous results (Fig. 2h) suggested implementing fermentation conditions that enhance acyl-CoA synthesis as well as its conversion to alka(e)nes rather than triglycerides. The optimization should also attempt to maximize cell biomass but not to the detriment of alka(e)ne synthesis. To this end, the following important process parameters were considered: (a) light intensity, which influences the efficiency of decarboxylation, (b) $\mathrm{C} / \mathrm{N}$ ratio and glucose concentration, which impact the extent of acyl-CoA synthesis flux, and (c) switching time from dark to light conditions, which affects the transition from biomass accumulation to alka(e)ne synthesis.

In previous studies, the yield of pentadecane from decarboxylation of palmitic acid in vitro increased linearly with light intensity ${ }^{11}$. However, it is unclear whether increasing light intensity would have the same effect in vivo due to possible issues with light penetration in a microbial culture. We, therefore, investigated two light sources with similar emission spectra (Supplementary Fig. 8a) but different intensities (Supplementary Fig. 8b). Between the two, light source 2 with higher intensity enhanced alka(e)ne production regardless of cell density (Supplementary Fig. 8c, d), suggesting that light penetration in the tested culture was not an issue. In line with a previous report ${ }^{14}$, we also observed that a much higher proportion of C17:1, C17:2, C15:0, and C15:1 was produced under higher light intensity (Supplementary Fig. 8c), indicating that the enzymatic specificity of $C v \mathrm{FAP}$ was light intensity dependent. The maximum titer reached $110 \mathrm{mg} / \mathrm{L}$ using the higher intensity light and a favorable $\mathrm{C} / \mathrm{N}$ ratio. The optimal $\mathrm{C} / \mathrm{N}$ ratio was determined to be approximately 43 , which perfectly balances the trade-off between more resources allocated to alka(e)ne synthesis versus more biomass to house the alka(e)nes (Supplementary Fig. 8d). Additionally, since there is little information available regarding the timing of light activation, we studied extensively how the onset of light exposure affects the culture (Supplementary Fig. 9). Cultivation without an initial dark phase showed the slowest growth and glucose consumption as well as the lowest alka(e)ne production (Supplementary Fig. 9a). Thus, we allowed the cells to grow and accumulate biomass under dark conditions prior to activating alka(e)ne synthesis. It was determined that cultivating the cells in the dark for 2 days followed by 1 day in the light was the optimal condition $(113 \mathrm{mg} / \mathrm{L})$, with other light patterns yielding poorer performance (Supplementary Fig. 9).

High initial glucose concentrations inhibit cell growth, substrate uptake, and lipid synthesis (Supplementary Fig. 10), in agreement with a previous report ${ }^{12}$. We thus investigated a fedbatch fermentation mode that allowed us to maintain relatively low glucose levels (Fig. 4a). Alka(e)ne titers increased by around $75 \mathrm{mg} / \mathrm{L} /$ day in the first 10 days where sufficient glucose was present. The production rate then slowed down afterward to about $50 \mathrm{mg} / \mathrm{L} / \mathrm{d}$, yielding a final alka(e)ne titer of $1.03 \mathrm{~g} / \mathrm{L}$ after 17 days. We examined the effect of $\mathrm{C} / \mathrm{N}$ ratio in the feeding media and found that feeding without nitrogen produced less alka(e)nes than those with nitrogen supplementation (Supplementary Fig. 11). This suggested that the $\mathrm{C} / \mathrm{N}$ ratios of both the initial and feeding media should be well controlled. Following the same feeding strategy, alka(e)ne titers of 1.20 and $1.47 \mathrm{~g} / \mathrm{L}$ were obtained from 120 and $160 \mathrm{~g} / \mathrm{L}$ glucose, respectively (Fig. $4 \mathrm{~b}$ ), which are the highest reported so far in yeast. 

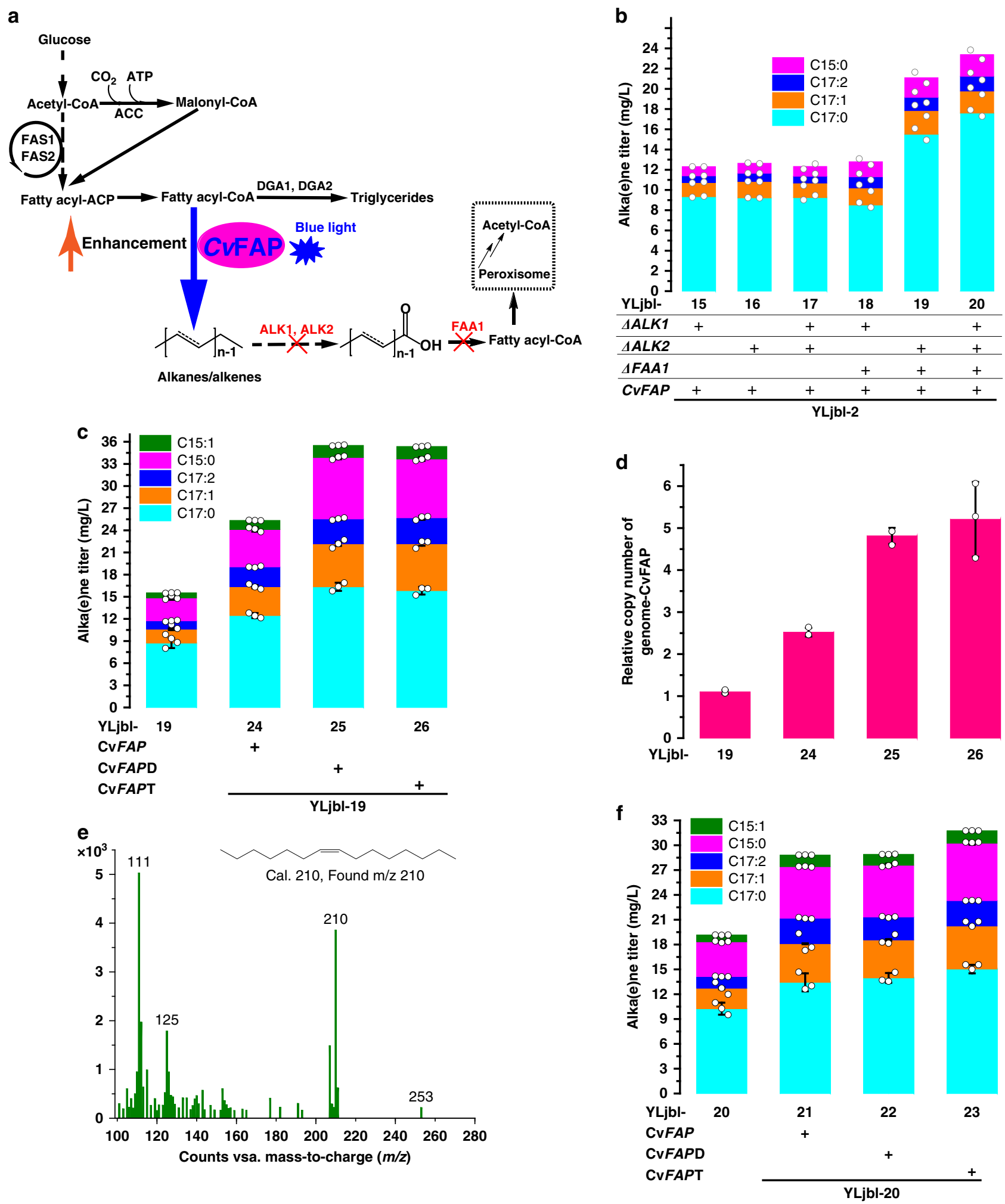

Our data showed that the amount of lipids in cells cultured in dark conditions matched the sum of lipids and alka(e)nes in cells cultured in light conditions (Fig. 4c), which suggests redirection of acyl-CoAs from lipids to alka(e)nes. Accordingly, we further developed a method (Supplementary Note 2) to determine the fraction of the redirected flux. This fraction increased from $31 \%$ to $85 \%$ as fermentation progressed from day 3 to 11 , asymptotically reaching a maximum of $89 \%$ (Fig. $4 \mathrm{~d}$, Supplementary Fig. 12), providing evidence that the majority of acyl-
CoA was rewired towards alka(e)ne synthesis during the light period.

We also examined the subcellular localization of the formed alka(e)nes. We first confirmed that alka(e)nes can fluoresce when emulsified in water with Tween 80 and stained with Nile red (Supplementary Fig. 13). Staining our alka(e)ne-producing strain reveals that most cells were filled with intracellular lipids and alka (e)nes with no evidence of extracellular fluorescence (Fig. 4e, f). Assuming that with further optimizations, the intracellular 
Fig. 3 Increasing CvFAP copy number debottlenecked the pathway for alka(e)ne production. a Metabolic pathways for improving alka(e)ne production by increasing CVFAP copy number. Abbreviations: ACC, acetyl-CoA carboxylase; FAS1 and FAS2, fatty acid synthase 1 and 2; DGAs, diacylglycerol transferases; ALK1 and ALK2, cytochrome P450ALKs belonging to the CYP52 family with major functions in n-alkane assimilation; 46 FAA1 was found to be related to $n$-alkane assimilation ${ }^{19}$. b Overexpression of CVFAP in strains with double deletions of FAA1 and ALK2 and triple deletions of FAA1, ALK1, and ALK2 enhanced alka(e)ne synthesis more profoundly ( $n=2$ biologically independent samples). c Compared with the parental strain YLjbl-19, CVFAP, CVFAPD, and CVFAPT transformants showed 1.7, 2.4, and 2.4-fold higher alka(e)ne titers, respectively ( $n=3$ biologically independent samples). $\mathbf{d}$ Relative copy numbers of the CVFAP gene were quantified and showed a positive correlation with alka(e)ne titers $(n=3$ biologically independent samples). e In addition, pentadecene (C15:1) derived from palmitoleoyl-CoA was detected in these strains but not the parental strains (observed from at least three biologically independent samples). f Alka(e)ne production of CVFAP, CvFAPD, and CVFAPT transformants from YLjbl-20 was improved as well, but not as much as the transformants from YLjbl-19 ( $n=3$ biologically independent samples). Fermentations in (b) were performed in $50 \mathrm{~mL}$ glass conical shake flasks with a working volume of $13 \mathrm{~mL}$ and an initial $\mathrm{OD}_{600}$ of 0.1 . Samples were collected after 3 days of cultivation in the dark followed by 3 days in blue light generated by light source 1 (Supplementary Fig. 16). Other fermentations were performed in 12-well microplates with a working volume of $2 \mathrm{~mL}$ and an initial $\mathrm{OD}_{600}$ of 0.5 . Samples were collected after 2 days of cultivation in the dark at $30^{\circ} \mathrm{C}$ followed by 1 day of cultivation in blue light generated by light source 2 at $25^{\circ} \mathrm{C}$ (Supplementary Fig. 16). Fermentation medium was composed of $20 \mathrm{~g} / \mathrm{L}$ glucose, $6.9 \mathrm{~g} / \mathrm{L}$ yeast nitrogen base (without amino acids), and $1 \mathrm{~g} / \mathrm{L}$ yeast extract. Data represent mean value \pm SD. Source data underlying Fig. $3 b-d, f$ are provided as a Source Data file.

accumulation of alka(e)nes can reach the levels reported for lipids, this product sequestration could offer unique advantages in its downstream separation from the other fermentation components.

Finally, alka(e)ne toxicity was investigated for YLjbl-26. The addition of $1.6 \mathrm{~g} / \mathrm{L}$ alka(e)nes in the fermentation medium did not affect cell growth (Supplementary Fig. 14), suggesting no toxicity from exogenous sources. Cell viability of YLjbl-26 was compared between a culture performed in dark conditions and a culture performed in normal alka(e)ne-producing conditions $(86.18 \mathrm{mg} /$ $\mathrm{L}$ titer). Using trypan blue staining, no dead cells were found in either culture (Supplementary Fig. 15), indicating that intracellular alka(e)nes did not pose observable toxic effects to the host cell. There was also no interference to acyl chain synthesis from the presence of intracellular alka(e)nes, as indicated in Fig. 4c. Overall, it can be concluded that the inhibitory effects of sequestered alka(e)nes to $Y$. lipolytica are negligible.

Production of alka(e)nes from $\mathrm{CO}_{2}$-derived substrates. The products made with our strains are suitable for use as jet fuel $(\mathrm{C} 15-\mathrm{C} 19)^{26}$ and diesel (C9-C23) $)^{27}$, both of which are important for the aviation industry and heavy vehicle transport. However, to achieve net-zero GHG emissions in these sectors without potential competition with food supply, $\mathrm{CO}_{2}$-neutral feedstocks other than corn or sugar cane-sourced glucose must be investigated. We thus examined the compatibility of our strain with two other notable feedstocks, wheat straw hydrolysate and acetate, which are primary products of $\mathrm{CO}_{2}$ fixation by plants and acetogens ${ }^{28}$, respectively (Fig. 5a).

Fermentation of wheat straw enzymatic hydrolysate was carried out following the developed fed-batch fermentation (Fig. 5b). Compared to the fermentation of glucose, alka(e)ne titers increased at a slower rate due to lower glucose concentrations and the possible presence of inhibitors ${ }^{29}$. The final titer and yield after 12 days of fermentation reached $242.04 \mathrm{mg} / \mathrm{L}$ and 4.66 $\mathrm{mg} / \mathrm{g}$ (glucose+yeast extract), respectively, with a productivity of $24.20 \mathrm{mg} / \mathrm{L} / \mathrm{d}$. The percentage of acyl-CoAs redirected to alka(e) nes increased with fermentation time and reached more than $95 \%$ after 10 days of fermentation (Fig. 5c).

Conversion of acetate to alka(e)nes was also very promising. Due to the slower growth in the acetate medium, cells were cultured in the dark phase for 3 days prior to light exposure (Fig. 5d). The uptake of acetate was slower than that of glucose. Alka(e)ne production ceased after 14 days reaching a final titer of $318.70 \mathrm{mg} / \mathrm{L}$, equivalent to a yield of $5.72 \mathrm{mg} / \mathrm{g}$ (acetic acid+yeast extract) and productivity of $28.98 \mathrm{mg} / \mathrm{L} / \mathrm{d}$. Acetate thus outperforms wheat straw hydrolysates. The maximum fraction of acyl-
CoAs that was diverted to alka(e)nes reached $94 \%$ at the end of the fermentation (Fig. 5e).

\section{Discussion}

Sorigué et al. ${ }^{11}$ discovered the fatty acid photodecarboxylase $C \nu$ FAP in response to blue light, characterized its blue light dependence, and elucidated its crystal structure. The enzyme was later expressed in Y. lipolytica strains with different genotypes for alka(e)ne production ${ }^{14}$. However, the detailed effects of each step along the metabolic pathway for alka(e)ne synthesis were not thoroughly studied. Our work elucidated the necessary steps for in vivo alka(e)ne production and identified the preferred substrate for the $C v$ FAP, resulting in a modified metabolic pathway that enabled the redirection of large proportion of acyl-CoA (89\%) to alka(e)nes. Driven by the deeper understanding, our work enhanced the alka(e)ne titers substantially compared with previous study ${ }^{14}$. For example, expression of CvFAP in an FFAoverproducing strain (approx. $2 \mathrm{~g} / \mathrm{L}$ intracellular and $2.5 \mathrm{~g} / \mathrm{L}$ extracellular $\mathrm{FFAs}^{20}$ ) produced $58.7 \mathrm{mg} / \mathrm{L}$ alka(e)nes using optimized fed-batch strategy ${ }^{14}$. By comparison, our highest titer from fed-batch fermentation $(1.47 \mathrm{~g} / \mathrm{L})$ was 25 -time more than that obtained from the previous study ${ }^{14}$. The main reason for the significant enhancement was the finding that acyl-CoA was the substrate for $C \nu \mathrm{FAP}$ and the modified metabolic pathway thereof.

The proposed pathway (Fig. $2 \mathrm{~h}$ ) suggests that FFA is not necessary for alka(e)ne production, thus reducing the accumulation of this toxic intermediate in the cell ${ }^{16,20}$. This also indicates that the expression of thioesterases or lipases is not required. Additionally, with a large pool of acyl-CoAs naturally present in $Y$. lipolytica, the employed debottlenecking strategy by simply increasing the CvFAP copy number can be very effective and requires less pathway engineering, thus reducing the burden of expressing multiple genes and balancing other pathways.

Furthermore, there are additional advantages of the discovered pathway. Previous efforts in microbial alka(e)ne production focused on the cyanobacterial pathway where acyl-ACP/acyl-CoA needs to be reduced to its corresponding aldehyde prior to decarbonylation 7,30 . The biological reduction step requires reducing equivalents in the form of $\operatorname{NAD}(\mathrm{P}) \mathrm{H}^{7}$. Similarly, the $\mathrm{OleT}_{\mathrm{IE}} \mathrm{P} 450$ catalyzed decarboxylation reaction to form alkenes directly from FFAs also requires $\mathrm{NAD}(\mathrm{P}) \mathrm{H}$ as a cofactor ${ }^{31,32}$. By contrast, the engineered strains in our work use a photo-driven decarboxylase that relies on extracellular photons from blue light to produce alka(e)nes, bypassing the $\mathrm{NAD}(\mathrm{P}) \mathrm{H}$ requirement. This allows the decoupling of alka(e)ne production from these cofactors, conserving $\mathrm{NAD}(\mathrm{P}) \mathrm{H}$ for biomass and acyl chain synthesis that are oftentimes limited by $\mathrm{NAD}(\mathrm{P}) \mathrm{H}$ availability in 
a

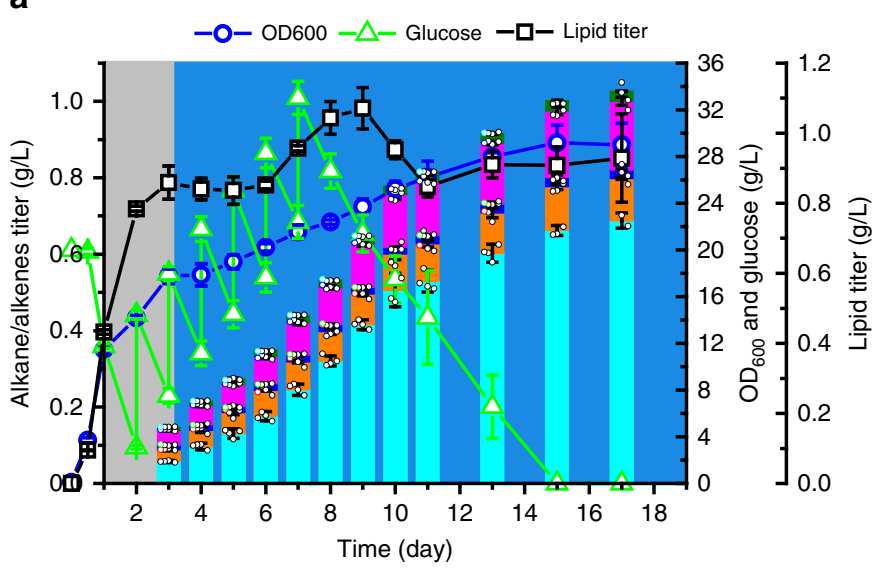

C

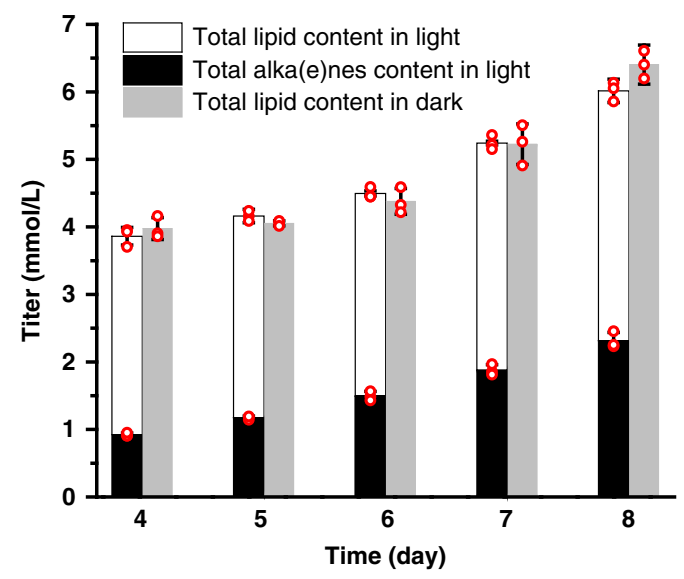

d

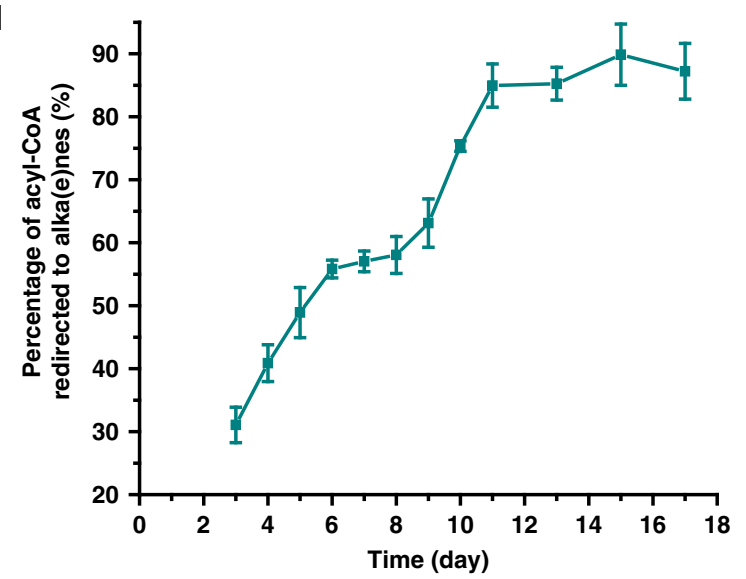

b

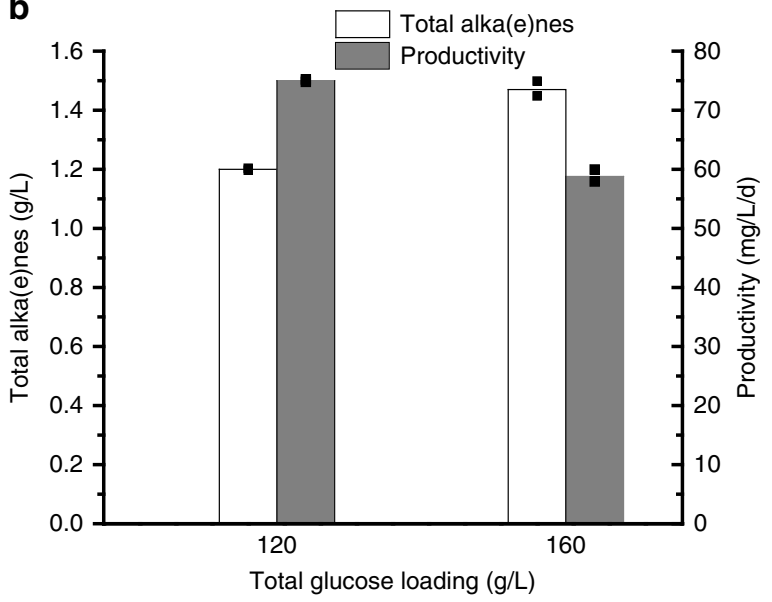

e

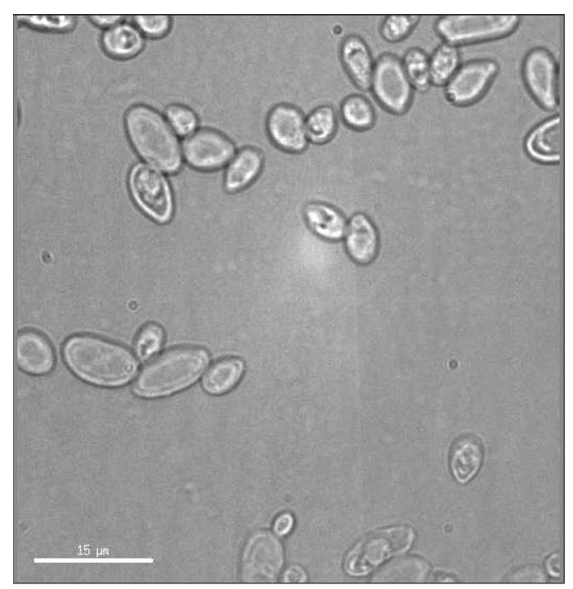

f

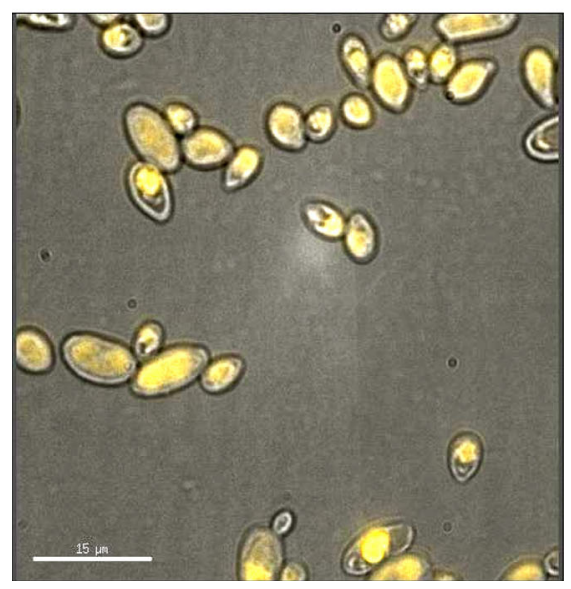

Fig. 4 Redirection of acyl-CoA to form alka(e)nes during fed-batch fermentation process. a Production of alka(e)nes by YLjbl-26 in fed-batch fermentations. Gray-colored regions indicate cultivation in the dark while blue-colored regions indicate cultivation in blue light ( $n=3$ or 4 biologically independent samples). b Alka(e)ne titers and productivities from different glucose loadings with the same feeding strategy as (a) ( $n=2$ biologically independent samples). c The amount of lipids in cells cultured in dark conditions matched the sum of lipids and alka(e)nes in cells cultured in light conditions. Both fermentations were carried out following the same feeding strategy as shown in (a). d We further developed a method (Supplementary Note 2) to determine the fraction of the redirected flux. This fraction increased from $31 \%$ to $85 \%$ as fermentation progressed from day 3 to 11 , asymptotically reaching a maximum of $89 \%$. e Brightfield microscopic images of cells with alka(e)ne titers of $1.03 \mathrm{~g} / \mathrm{L}$, equivalent to $89 \%$ of neutral lipids. f Fluorescence images of cells (yellow), merged with the brightfield images. Staining our alka(e)ne-producing strain reveals that most cells were filled with intracellular lipids and alka(e)nes with no evidence of extracellular fluorescence. Images were taken by a DeltaVision2-TIRF Microscope. Cells were stained by Nile red. Fermentations were performed in $50 \mathrm{~mL}$ glass conical shake flasks with a working volume of $13 \mathrm{~mL}$ and an initial $\mathrm{OD}_{600}$ of 0.1 . Blue light was generated by light source 2 (Supplementary Fig. 16). Data represent mean value $\pm S D, n=3$ or $n=4$ biologically independent samples. Source data underlying Fig. 4a-d are provided as a Source Data file. 
a

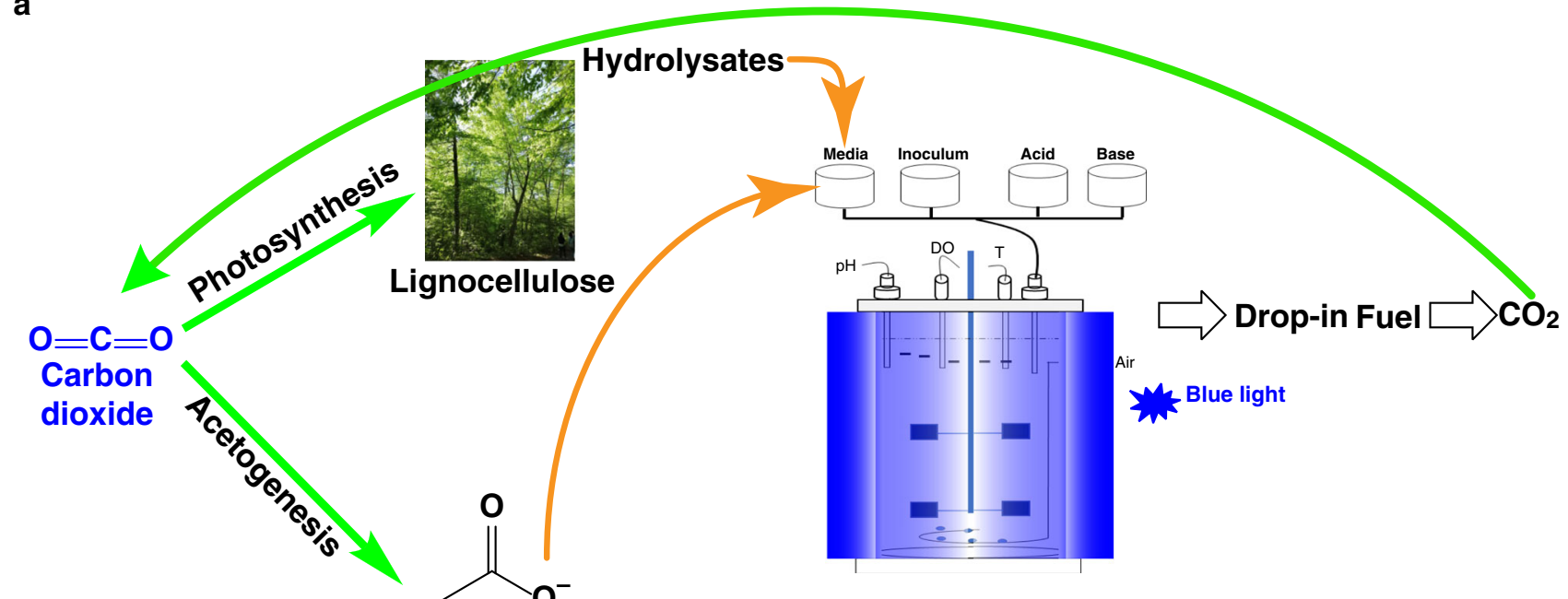

Acetate

b
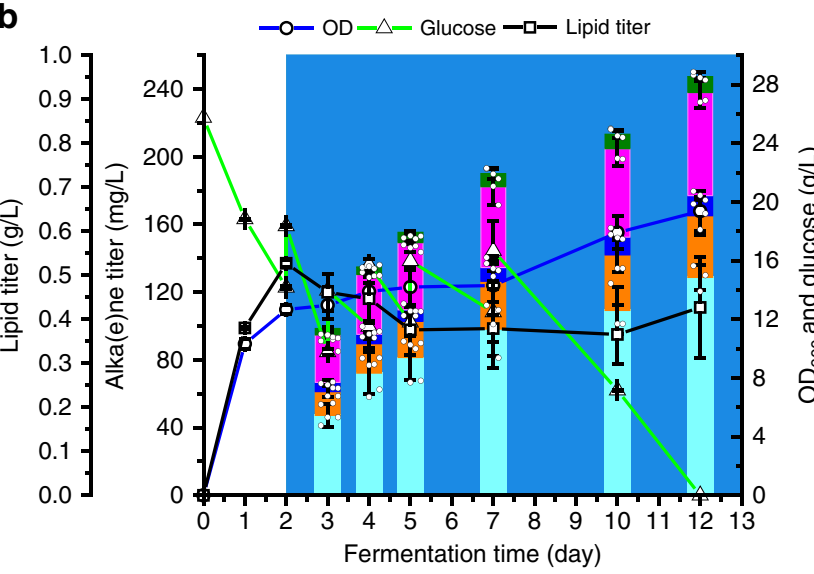

C

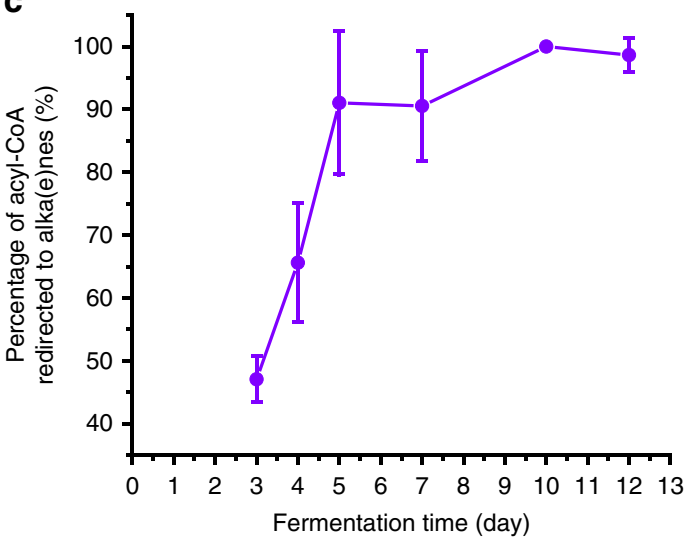

d

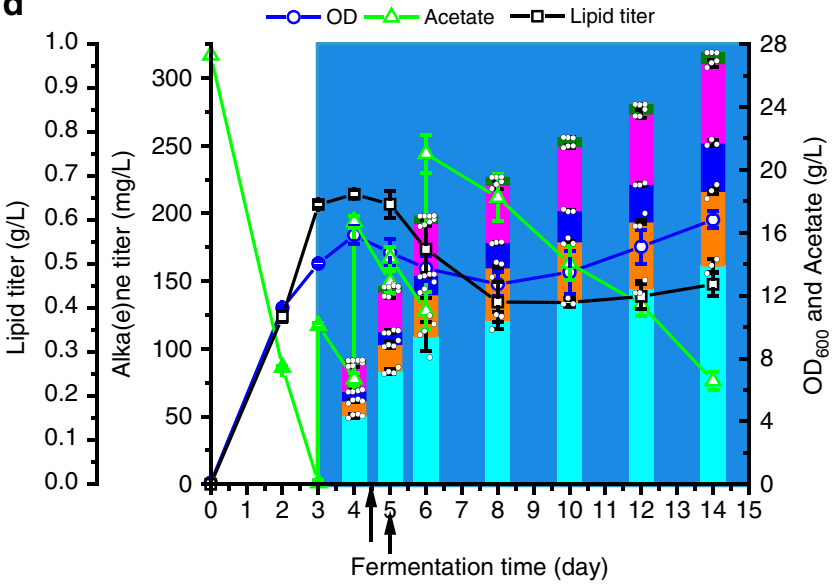

e

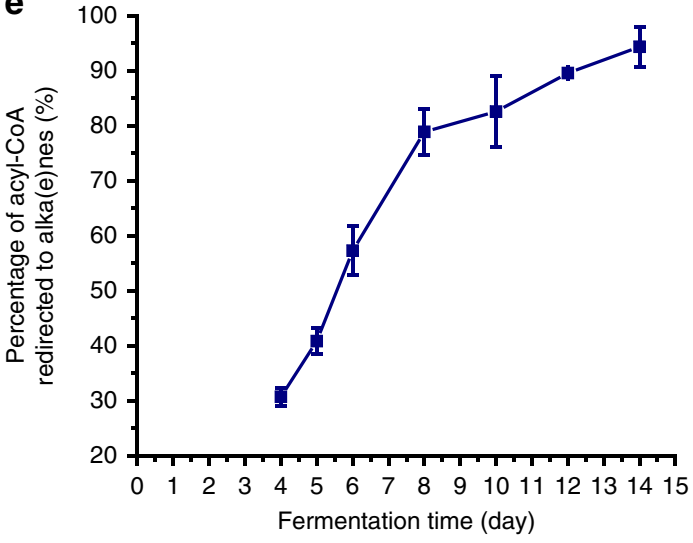

Fig. 5 Alka(e)ne production from two $\mathbf{C O}_{2}$-neutral substrates. a Schematic diagram to show how the strain and process developed in this work could advance the net-zero $\mathrm{GHG}$ emissions by using $\mathrm{CO}_{2}$-neutral feedstocks other than corn or sugar canes-sourced glucose. $\mathbf{b}$ Fermentation time course of alka (e)ne production from wheat straw hydrolysates. c The percentage of acyl-CoAs that were redirected to form alka(e)nes during wheat straw hydrolysate fermentation. $\mathbf{d}$ Fermentation time course of alka(e)ne production from sodium acetate. $\mathbf{e}$ The percentage of acyl-CoAs that were redirected to form alka (e)nes during acetate fermentation. Fermentations were performed in $50 \mathrm{~mL}$ glass conical shake flasks with a working volume of $13 \mathrm{~mL}$ and an initial $\mathrm{OD}_{600}$ of 0.1. Blue light was generated by light source 2 (Supplementary Fig. 16). Data represent mean value $\pm S D, n=3$ biologically independent samples. J. Li created the tree image in (a). Source data underlying Fig. $5 b-d$ are provided as a Source Data file. 
Y. lipolytica ${ }^{33}$. It is also noteworthy that alka(e)nes with specific composition may be tailored by altering the light intensity (Supplementary Fig. 8c) or engineering $C v \mathrm{FAP}^{14}$.

The endogenously formed alka(e)nes exhibited neglectable toxicity and were sequestered intracellularly (Fig. 4e, f), which may be facilitated by the intrinsic ability of Y. lipolytica to form lipid bodies ${ }^{34}$. Sequestering products inside cells offers simpler downstream processing for product recovery as there is no need to extract and separate products from the fermentation broth, significantly lowering the production cost. Overall, the benefits of alka(e)ne sequestration and the ability of Y. lipolytica to naturally drive more carbon flux to acyl-CoAs make it a preferred organism for alka(e)ne production.

With an initial glucose loading of $80 \mathrm{~g} / \mathrm{L}, 1.03 \mathrm{~g} / \mathrm{L}$ alka(e)nes and $0.93 \mathrm{~g} / \mathrm{L}$ lipids were produced, corresponding to a yield of approximately $24.5 \mathrm{mg} / \mathrm{g}$ glucose, which was comparable to that of a wild-type $Y$. lipolytica strain expressing $\beta$-galactosidase (23 $\mathrm{mg} / \mathrm{g})^{24}$. Though the comparable yield was achieved, we explored more of the carbon balance and found that apart from alka(e)nes and lipids, the major byproducts in the fermentation broth were determined to be $33.01 \mathrm{~g} / \mathrm{L}$ pyruvate and $2.74 \mathrm{~g} / \mathrm{L}$ citrate. Assuming similar yields of these products to that reported in the literature ${ }^{35-37}$, the measurements satisfy a closed carbon balance. The results also guide us that future works should be focused on directing the pyruvate flux to acyl-CoA to further enhance the alka(e)ne yield, which may be achieved by enhancing pyruvate dehydrogenase $^{38}$ or introducing pyruvate dehydrogenase bypass $^{39}$. Besides, increasing acetyl-CoA pool by feeding acetate as direct substrate ${ }^{39}$ following the substrate co-feeding strategy ${ }^{28}$ may also improve alka(e)ne titer. Overexpression of citrate shuttle consisting of ATP-citrate lyase ${ }^{40}$ may increase acetyl-CoA pool and thereby enhance alka(e)ne production as well.

By employing the engineered strain and the developed process, two $\mathrm{CO}_{2}$-derived substrates were converted to alka(e)nes effectively. Further engineering of YLjbl-26 to utilize xylose ${ }^{41}$ and tolerate inhibitors could increase the yield of alka(e)nes from lignocelluloses. With regards to acetate conversion, the energy efficiency of $\mathrm{H}_{2} / \mathrm{CO}_{2}$-to-acetate-to-lipids conversion can reach $38 \% 28$. Considering that $94 \%$ of acyl-CoAs were converted to alka (e)nes (Fig. 5e), we can estimate that a potential process generating alka(e)nes from $\mathrm{H}_{2} / \mathrm{CO}_{2}$ would have a theoretical efficiency of $35.7 \%$ if the co-feeding strategies ${ }^{28}$ were adopted. With further development of the conventional bioreactors equipped with internal blue light bulbs focusing on controlling the light intensity and enhancing penetration, the scale-up of either process to produce drop-in fuels with tailored composition would be possible. These fuels can be used directly in our existing infrastructure, transforming the current long-distance road transport, aviation, and shipping to a more sustainable one.

\footnotetext{
Methods

Strains and reagents. Y. lipolytica strains and plasmids used are listed in Supplementary Tables 2 and 3. KAPA HiFi DNA Polymerase with high-fidelity was purchased from KapaBiosystems. GoTaq Green Master Mix was purchased from Promega Corp and used for colony PCR. Gibson assembly, chemical competent $E$. coli cell (DH 5a), and restriction enzymes were purchased from New England Biolabs. DNA gel recovery and plasmid extraction kits were purchased from Qiagen. All heterologous genes were codon-optimized and synthesized by ThermoFisher Scientific. All primers were synthesized by MilliporeSigma. Hexane, methanol, $\mathrm{H}_{2} \mathrm{SO}_{4}, \mathrm{NaOH}$, and dodecane were purchased from MilliporeSigma. Yeast extract and peptone were from Bacto. The nitrogen content of the yeast extract was $11 \%(\mathrm{w} / \mathrm{w})$. Yeast nitrogen base without amino acids and ammonium sulfate (YNB-AA-AS-) was from VWR Life Science. Alkane standards were purchased from VWR with purity higher than $98 \%$.

Wheat straw was pretreated according to the previous work ${ }^{42}$. Briefly, $50 \mathrm{~g}$ wheat straw was cut to pieces with the average length of roughly $0.5 \mathrm{~cm}$ and added to $500 \mathrm{~mL}$ of $2 \% \mathrm{NaOH}$ aqueous solution to a final solid concentration of $10 \%$. The mixture was subsequently subjected to $121^{\circ} \mathrm{C}$ for $1 \mathrm{~h}$ and filtered through a 20 mesh $(0.85 \mathrm{~mm})$ filter to collect the solids. The solids were then washed to reach
}

neutral $\mathrm{pH}$, concentrated by pressing water out, and yielded around $140 \mathrm{~g}$ pretreated wheat straw with water content of approximately $75 \%$. This mixture was directly hydrolyzed by adding $10 \mathrm{~mL}$ of citrate buffer ( $\mathrm{pH} 5.5$ ) and $2 \mathrm{~mL}$ Cellic CTec2 (Sigma-Aldrich) for 4 days at $50^{\circ} \mathrm{C}$ and $250 \mathrm{rpm}$. The final glucose concentration was $83.8 \mathrm{~g} / \mathrm{L}$ in the hydrolysate.

Bjour Curtain String Lights 300 LED Window Curtain Icicle Blue Light manufactured by Twinkle Star was purchased from Amazon (Light source 1). Water-Resistance IP65, 12 V Waterproof Flexible LED Strip Light, $16.4 \mathrm{ft} / 5 \mathrm{~m}$ Cuttable LED Light Strips, 300 Units 2835 LEDs Lighting String manufactured by Tasodin was purchased from Amazon (Light source 2). The LED power was measured with a PD300-3W photodiode sensor (Ophir Photonics, https://www ophiropt.com/laser-measurement/laser-power-energy-meters/products/LaserPhotodiode-Sensors/Standard-Photodiode-Sensors/PD300-3W) set at the LED peak wavelength connected to a Vega power meter (Ophir Photonics, https://www. ophiropt.com/laser-measurement/laser-power-energy-meters/products/smartdisplays/vega).

Genetic manipulation. Gene fragments were either synthesized as shown in Supplementary Data 1 or amplified with primers listed in Supplementary Table 5. Gibson Assembly was used to ligate the fragments into ring form plasmids. Transformation of the resultant mixture into E. coli $\mathrm{DH} 5 a$ by heat shock at $42{ }^{\circ} \mathrm{C}$ for $1 \mathrm{~min}$, followed by plating cells on Petri dishes with LB agar containing corresponding antibiotics were performed. Colonies were checked with colony PCR using primers on promoter and structural gene to select positive ones. All plasmids harboring one cassette were constructed by the above-described procedures. Plasmids harboring repeated pTef-in-CvFAP-XPR2t cassette were constructed by the single restriction enzyme digestion, followed by Gibson Assembly. Plasmids were extracted from the cultivated positive strains and sequenced. All plasmids used in this work are listed in Supplementary Table 2.

All plasmids were linearized by restriction enzyme NotI or AseI before transformation into auxotrophic Y. lipolytica $\left(U R A 3^{-}\right)$. The transformation medium was composed of $2.25 \mathrm{~mL}$ of $50 \%$ PEG 3350 in water, $0.125 \mathrm{~mL} 2 \mathrm{M}$ lithium acetate, and $0.125 \mathrm{~mL} 2 \mathrm{M}$ DTT in water. Transformation medium of 100 $\mu \mathrm{L}, 5 \mu \mathrm{L}$ of single-strand DNA $(10 \mathrm{~g} / \mathrm{L})$, and plasmid DNA of interest $(1 \mu \mathrm{g})$ were mixed together with the auxotrophic parent strain, vortexed for $15 \mathrm{~s}$, and placed in $28^{\circ} \mathrm{C}$ for $30 \mathrm{~min}$, followed by incubating at $39^{\circ} \mathrm{C}$ for $30 \mathrm{~min}$. The resultant mixture was spread on YNB-Uracil ${ }^{-}$agar plate (see below for the composition) and allowed to be in $30^{\circ} \mathrm{C}$ incubator for $2-3$ days. Recombinants were checked by colony PCR and the positive ones were selected for the first-round screening fermentation.

Gene knockouts were performed according to the developed CRISPR method ${ }^{43}$ Briefly, gRNA sequences were designed by Benchling with NGG as PAM.

Homologous arms with $100 \mathrm{bps}$ composed of $50 \mathrm{bps}$ from the upstream and $50 \mathrm{bps}$ from downstream were used to delete the part in between. The positive knockouts were examined by PCR with genomic DNA as templates. All primers, homologous arms, and gRNA sequences related to gene knockouts are listed in Supplementary Table 4.

After each transformation or knockout, colony PCR-positive Y. lipolytica strains were further screened by microplate fermentation. Briefly, 12 positive strains were inoculated to 12 -well microplate containing $2 \mathrm{~mL}$ YEM medium in each well and cultivated in dark at $30^{\circ} \mathrm{C}, 225 \mathrm{rpm}$ for 2 days, followed by cultivation in blue light at $25^{\circ} \mathrm{C}, 150 \mathrm{rpm}$ for 1 day prior to alka(e)ne analyses. Strains with the highest alka (e)ne titer were selected for further studies.

Counterselection against 5 -Fluoroorotic acid (5-FOA) was performed to recover URA3 marker. Briefly, gene fragment of $1000 \mathrm{bp}$ containing both upstream and downstream of URA3 was transformed into the Y. lipolytica strains of interest, followed by spreading on yeast nitrogen base medium (YNB-FOA) containing 1 $\mathrm{mg} / \mathrm{mL} 5$-FOA and $5 \mathrm{mg} / \mathrm{mL}$ uracil. The colonies that grew over 3 days were transferred onto an uracil-minus plate and a corresponding YPD plate to confirm the auxotroph.

Media and conditions. Luria-Bertani (LB) medium containing $10 \mathrm{~g} / \mathrm{L}$ peptone (Bacto), $5 \mathrm{~g} / \mathrm{L}$ yeast extract (Bacto), $10 \mathrm{~g} / \mathrm{L} \mathrm{NaCl}$ (Sigma), and corresponding antibiotics (carbenicillin $100 \mu \mathrm{g} / \mathrm{mL}$, kanamycin $50 \mu \mathrm{g} / \mathrm{mL}$, or chloramphenicol 34 $\mu \mathrm{g} / \mathrm{mL}$ ) was used to culture E. coli harboring plasmid. Culturing tubes with the inoculated medium was placed on a rotatory drum at $37^{\circ} \mathrm{C}$ for around $15 \mathrm{~h}$ for plasmid proliferation.

Different solid media were prepared as follows. YNB-Uracil' ${ }^{-}$medium composed

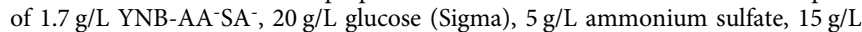
agar (Bacto), with an appropriate supplement of $0.77 \mathrm{~g} / \mathrm{L}$ complete supplement mixture minus uracil (Sunrise science products) was used for selecting transformed Y. lipolytica strains. YNB-FOA medium containing $1.7 \mathrm{~g} /{\mathrm{L} \mathrm{YNB}-\mathrm{AA}^{-} \mathrm{SA}}^{-}, 5 \mathrm{~g} / \mathrm{L}$ ammonium sulfate, $20 \mathrm{~g} / \mathrm{L}$ glucose, $0.77 \mathrm{~g} / \mathrm{L}$ complete supplement mixture and $1 \mathrm{~g} /$ L 5-fluoroorotic acid (Zymo Research) was used to counterselect the URA3disrupted strains. YPD medium containing $10 \mathrm{~g} / \mathrm{L}$ yeast extract, $20 \mathrm{~g} / \mathrm{L}$ peptone, 20 $\mathrm{g} / \mathrm{L}$ glucose was used to culture auxotrophic strains.

For alka(e)ne fermentation before process optimization, YEM medium

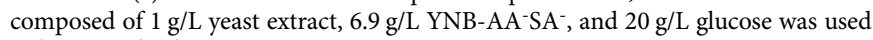
unless stated otherwise. 
Batch fermentation. Batch fermentations for alka(e)ne production were performed in either $50 \mathrm{~mL}$ conical glass flasks or Falcon polystyrene 12 well microplates for either 3 days or 2 days in dark followed by 3,2, or 1 day in blue light. The temperature was controlled at $30^{\circ} \mathrm{C}$ in dark while $25^{\circ} \mathrm{C}$ in light. The light sources with a maximum peak wavelength of 454 and $458 \mathrm{~nm}$ (Supplementary Fig. 8a) were applied during light cultivation phase. The working volume for $50 \mathrm{~mL}$ conical glass flasks and 12 well plates was $13 \mathrm{~mL}$ and $2 \mathrm{~mL}$, respectively with inoculum of the initial $\mathrm{OD}_{600}$ of 0.1 and 0.5 , respectively. The self-made instrument for cultivation in blue light is shown in Supplementary Fig. 16.

Batch fermentations with supplementation of palmitic acid- $\mathbf{d}_{\mathbf{3 1}}$. Fermentations were carried out by using strains YLjbl-2 and YLjbl-2- $\triangle F A A 1$. Medium containing

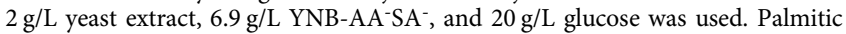
acid- $\mathrm{d}_{31}$ (dissolved in absolute ethanol) with a final concentration of $5 \mathrm{~g} / \mathrm{L}$ was added before switching to blue light cultivation. In control experiments, the same amount of absolute ethanol was added.

Fed-batch fermentation with glucose. Medium containing $2 \mathrm{~g} / \mathrm{L}$ yeast extract,

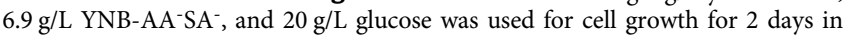
dark. Thereafter, glucose or glucose with different amounts of yeast extract composed of $200 \mathrm{~g} / \mathrm{L}$ glucose and $5,10,15 \mathrm{~g} / \mathrm{L}$ yeast extract, equivalent to a $\mathrm{C} / \mathrm{N}$ ratio of 170,85 , and 57 was fed every $24 \mathrm{~h}$ until the total glucose reached 80,120 , and 160 $\mathrm{g} / \mathrm{L}$. From day 2 to day 7 , the feeding medium was supplemented to increase the glucose concentration by $10 \mathrm{~g} / \mathrm{L}$ each day. $\mathrm{pH}$ was not controlled. All fed-batch fermentations were carried out in $50 \mathrm{~mL}$ conical flasks with a working volume of $13 \mathrm{~mL}$ for 2 days in dark followed by culture in blue light generated by light source 2 at $25^{\circ} \mathrm{C}$ (Supplementary Fig. 16). The initial $\mathrm{OD}_{600}$ was 0.1 and feeding was conducted when the increase in glucose/acetate could be observed in Figs. 4 and 5.

Fed-batch fermentation with acetate. For fed-batch fermentation with acetate,

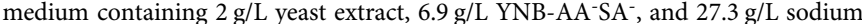
acetate was used for cell growth for 3 days in dark. Acetic acid was added at 4.5 days and 5 days to adjust $\mathrm{pH}$ to 6.8 . Sodium acetate $(273 \mathrm{~g} / \mathrm{L}$ with $5 \mathrm{~g} / \mathrm{L}$ yeast extract) was used as a feeding medium. The acetic acid added was $2.31 \mathrm{~g} / \mathrm{L}$ and in total $57.3 \mathrm{~g} / \mathrm{L}$ acetic acid equivalence was added. Yeast extract of $2.75 \mathrm{~g} / \mathrm{L}$ was added during the whole fermentation process. For stabilizing pH, $5 \mathrm{mM}$ PBS ( $\mathrm{pH}$ 6.0) was used at the initial stage and $\mathrm{HCl}(6 \mathrm{M})$ was used to control $\mathrm{pH}$ around 6.8 every $24 \mathrm{~h}$

Fed-batch fermentation with wheat straw hydrolysate. For fed-batch fermentation with wheat straw hydrolysate, medium containing $1 \mathrm{~g} / \mathrm{L}$ yeast extract

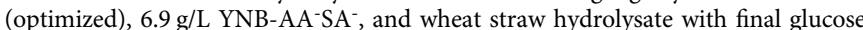
of $25.74 \mathrm{~g} / \mathrm{L}$ was used for cell growth for 2 days in dark. The hydrolysate with a glucose concentration of $83.8 \mathrm{~g} / \mathrm{L}$ without yeast extract supplement was used as feeding medium for 6 times. The total glucose and yeast extract added were $50.89 \mathrm{~g} /$ $\mathrm{L}$ and $1 \mathrm{~g} / \mathrm{L}$ yeast extract, respectively, during the whole fermentation process without controlling the $\mathrm{pH}$.

Analytical methods for metabolites. $\mathrm{OD}_{600}$ was measured by a spectrophotometer against corresponding blanks. Dilutions were carried out to make sure the $\mathrm{OD}_{600}$ value ranging from 0.1 to 0.9 .

Glucose and acetic acid in the supernatant of cultures were analyzed by highperformance liquid chromatography (HPLC, Agilent Technologies, 1260 Infinity) equipped with a BioRad HPX-87H column (BioRad) and a refractive index detector (Agilent Technologies, 1260 Infinity). External standard curves using corresponding compounds with analytical grades were prepared for quantification purposes. Agilent OpenLab Software 7890B was used to collect and analyze data.

For alkane/alkene quantification, cell pellets from 0.05 to $1 \mathrm{~mL}$ cell culture depending on the content were collected by centrifuge at $18,406.75 \times g$ for $5 \mathrm{~min}$. Sodium hydroxide-methanol solution $(0.5 \mathrm{~mol} / \mathrm{L})$ of $0.5 \mathrm{~mL}$ was mixed with cell pellets, followed by adding internal standards, glyceryl triheptadecanoate (Sigma, 2 $\mathrm{g} / \mathrm{L}$ ) and dodecane (Sigma, $37.5 \mathrm{mg} / \mathrm{L}$ ) in hexane. The mixture was sonicated for 15 $\mathrm{min}$ and vortexed (Vortex-Genie 2, Scientific Industries) for $1 \mathrm{~h}$ at room temperature $\left(24 \pm 2{ }^{\circ} \mathrm{C}\right)$ prior to being acidified by adding $60 \mu \mathrm{L}$ condensed $\mathrm{H}_{2} \mathrm{SO}_{4}$ (Sigma) with caution. Hexane $(0.5 \mathrm{~mL})$ was added and vortexed for $30 \mathrm{~min}$ to extract fatty acid methyl esters and alkanes/alkenes. External standard curves were prepared by running the same procedure using corresponding standard compounds of analytical grade. The upper phase after centrifugation was analyzed by GC-MS (Agilent Technologies, 7890B Series GC and a 5977B MS) or GC-FID (Agilent 7890B). For GC-MS, $3 \mu \mathrm{L}$ of extract in hexane was injected without split into an HP-5MS UI column (Agilent Technologies). Whereas for GC-FID, $1 \mu \mathrm{L}$ of extract in hexane was injected with the split ratio of 2:1 into an HP-INNOWAX column (Agilent Technologies). For GC-FID, the inlet and FID temperature was held at $260^{\circ} \mathrm{C}$ using the following temperature program: initial $2 \mathrm{~min}$ at $40^{\circ} \mathrm{C}$, then ramped to $200{ }^{\circ} \mathrm{C}$ at a rate of $20^{\circ} \mathrm{C} / \mathrm{min}$, held for $2 \mathrm{~min}$, and ramped to $250{ }^{\circ} \mathrm{C}$ at a rate of $40^{\circ} \mathrm{C} / \mathrm{min}$, and held for $5 \mathrm{~min}$. For GC-MS, the inlet, transfer tube, and MS source temperatures were set at 320,300 , and $280^{\circ} \mathrm{C}$, respectively. The MS was operated in scan mode from 100 to $450 \mathrm{~m} / z$. The temperature program was: initial 2 min at $40^{\circ} \mathrm{C}$, then ramped to $270{ }^{\circ} \mathrm{C}$ at a rate of $12^{\circ} \mathrm{C} / \mathrm{min}$, and ramped to $310^{\circ} \mathrm{C}$ at a rate of $6^{\circ} \mathrm{C} / \mathrm{min}$, and held for $10 \mathrm{~min}$. The standard curves of different alka(e) nes were shown in Supplementary Fig. 17. Agilent OpenLab Software 7890B was used to collect data.

For FFA quantification, $0.5 \mathrm{~mL}$ culture was mixed with $80 \mu \mathrm{L}$ glass beads, 150 $\mu \mathrm{L}$ methanol, and $150 \mu \mathrm{L}$ hexane containing pentadecanoic acid as internal standard. The mixture was rigorously vortexed for $2 \mathrm{~h}$, followed by centrifugation to facilitate phase separation. The upper hexane phase was taken for GC-MS analysis. The inlet, transfer tube, and MS source temperatures were set at 320,300 , and $280^{\circ} \mathrm{C}$, respectively. The MS was operated in scan mode from 150 to $500 \mathrm{~m} / \mathrm{z}$. The temperature program was: initial at $100{ }^{\circ} \mathrm{C}$, then ramped to $270{ }^{\circ} \mathrm{C}$ at a rate of $12^{\circ} \mathrm{C} / \mathrm{min}$, and ramped to $310^{\circ} \mathrm{C}$ at a rate of $6^{\circ} \mathrm{C} / \mathrm{min}$, and held for $10 \mathrm{~min}$. Helium was used as a carrier gas.

Substrate docking and structural analysis. Crystal structure images were produced by using the UCSF Chimera package from the Computer Graphics Laboratory, University of California, San Francisco (supported by NIH P41 RR01081) ${ }^{44}$. Substrate docking was performed by using AutoDock Vina 1.1.2, with implementation through Chimera 1.13.1 $\mathrm{rc}^{45}$. Protein structure (with fatty acid substrate and FAD deleted) and fatty acyl-CoA substrate were prepared by using the DockPrep tool of Chimera. Analysis of the resulting docking modes was performed through the ViewDock tool, which listed 10 docking modes according to their energy scores. The best docking model was decided by a combination of criteria: visual inspection (correct orientation of the fatty acid and nucleotide ends of acyl-CoA, match of the nucleotide binding site) and energy scores.

\section{Quantitative real-time PCR and reverse transcription-qPCR analyses. For} quantitative real-time PCR, total genomic DNA extracted from each individual strains of interest by using the Phenol-Chloroform protocol was used as template For reverse transcription-qPCR, mRNA extracted by MasterPure ${ }^{\mathrm{TM}}$ Yeast RNA purification kit (Lucigen, Wisconsin, USA) was used as template. PCRs were performed using iScript ${ }^{\mathrm{TM}}$ one-step RT-PCR kit with SYBR $^{\circ}$ green (Bio-Rad) with the designed primers of $\mathrm{CvFAP}$ and internal reference ACT (Supplementary Table 5) on an iCycler (Bio-Rad), according to the manufacturer's instructions. Relative gene expression was performed using the comparative $2^{-\Delta \Delta \mathrm{Ct}}$ or $2^{-\Delta \mathrm{Ct}}$ method.

CuFAP protein expression and purification. The construct consisting of the CvFAP gene with an optimized codon for Y. lipolytica and a sequentially $6 \times$ His tag on 3'-terminus were cloned into a pET28 vector backbone using Gibson Assembly. The plasmid was then transformed in E. coli DH5a and the positive colonies were identified by colony-PCR. Three plasmids were extracted and sequenced to select the correct one for protein expression.

The selected plasmid was transformed in E. coli BL21 (DE3) (purchased from $\mathrm{NEB}$ ). The recombinant was precultured in $5 \mathrm{~mL} \mathrm{LB}$ medium containing $50 \mu \mathrm{g} / \mathrm{L}$ kanamycin overnight. The preculture was then inoculated to $500 \mathrm{~mL}$ medium with the same composition as the preculturing medium. IPTG was added with the final concentration of $0.5 \mathrm{mM}$ when the $\mathrm{OD}_{600}$ reached $0.8-0.9$. The culture was then moved to an incubator at $16^{\circ} \mathrm{C}$ and $160 \mathrm{rpm}$ for $20 \mathrm{~h}$. Cells were harvested by centrifugation at $4{ }^{\circ} \mathrm{C}$ and the cell pellet was washed with Tris- $\mathrm{HCl}$ buffer $(50 \mathrm{mM}$, $\mathrm{pH} 8.5)$ once.

The cell pellet was resuspended by NPI-10 for cell disruption. The suspended cells were disrupted by a sonic disruptor on ice for $30 \mathrm{~min}$. Protein purification was carried out according to the protocol (https://www.mn-net.com/Portals/8/ attachments/Redakteure_Bio/Protocols/Protino/UM_Protino96NTA.pdf). The resultant protein fractions were examined by SDS-PAGE as shown in Supplementary Fig. 18. Fractions from NPI-125 and NPI-250 were combined for following buffer change. Buffer change was performed by washing NPI-125 and NPI-250 fractions through $10 \mathrm{kD}$ cut-off using Tris- $\mathrm{HCl}$ buffer $(50 \mathrm{mM}, \mathrm{pH} 8.5)$ for 3 times. The obtained protein was used for in vitro photodecarboxylation experiments.

In vitro photocatalytic reactions. The reactions using stearoyl-CoA lithium salt (Sigma) and stearic acid lithium salt as substrates were performed at $25^{\circ} \mathrm{C}$ in a total volume of $100 \mu \mathrm{L}$. Different volumes of substrates in DMSO $(0.97 \mathrm{mM})$, a fixed amount of purified enzyme $(20 \mu \mathrm{L}, 3.30 \mathrm{mg} / \mathrm{mL})$, pure DMSO, and Tris- $\mathrm{HCl}$ buffer $(50 \mathrm{mM}, \mathrm{pH} 8.5)$ were added in transparent glass vials $(2 \mathrm{~mL})$ with final DMSO of $30 \mathrm{vol} \%$. The vials were screw-capped and placed in the shaker with blue light for $20 \mathrm{~h}$ at $150 \mathrm{rpm}$. The product was extracted with ethyl acetate and analyzed by the early-mentioned GC-FID method.

Cell viability measurements. To measure cell viability, Trypan blue (SigmaAldrich, $0.4 \%$ in water) was added directly to aliquots of undiluted cultures with a final concentration of $0.2 \%$ and visualized immediately at $1000 \times$ magnification on a ZEISS Axioskop (West Germany) by bright field microscope. Images were recorded using a Nikon color camera.

Staining measurements of alka(e)nes and cells containing alka(e)nes. An artificial mixture of $10 \mathrm{mg}$ of alka(e)nes containing C17:0, C17:1 (terminal double 
bond), and C15:0 was mixed with $0.5 \mathrm{~mL}$ water and $100 \mu \mathrm{L}$ Tween 80 . The mixture was vigorously vortexed at $1000 \mathrm{rpm}$ for $5 \mathrm{~min}$ before being stained by Nile red (Ethanol solution, $0.1 \mu \mathrm{g} / \mathrm{ml}$ ). Images were taken by using a DeltaVision2-TIRF Microscope.

Reporting summary. Further information on research design is available in the Nature Research Reporting Summary linked to this article.

\section{Data availability}

The data supporting the findings of this study are available within the paper and its supplementary information files. A reporting summary for this article is available as a supplementary information file. The datasets and materials generated and analyzed during the current study are available from the corresponding author upon request. Structure of fatty acid photodecarboxylase in complex with FAD and palmitic acid is available from Protein Data Base with ID 5NCC (DOI: 10.2210/pdb5NCC/pdb) [https:// www.rcsb.org/structure/5NCC]. Source data are provided with this paper.

Received: 17 June 2020; Accepted: 8 November 2020;

Published online: 03 December 2020

\section{References}

1. Liao, J. C., Mi, L., Pontrelli, S. \& Luo, S. Fuelling the future: microbial engineering for the production of sustainable biofuels. Nat. Rev. Microbiol. 14, 288-304 (2016).

2. Davis, S. J. et al. Net-zero emissions energy systems. Science 360, eaas9793 (2018).

3. Hong, T. D. et al. A study on developing aviation biofuel for the Tropics: production process-experimental and theoretical evaluation of their blends with fossil kerosene. Chem. Eng. Process. 74, 124-130 (2013).

4. Zhou, Y. J., Kerkhoven, E. J. \& Nielsen, J. Barriers and opportunities in biobased production of hydrocarbons. Nat. Energy 3, 925-935 (2018).

5. Xin, J. et al. Formation of $\mathrm{C}-\mathrm{C}$ bonds for the production of bio-alkanes under mild conditions. Green. Chem. 16, 3589-3595 (2014).

6. Clomburg, J. M., Crumbley, A. M. \& Gonzalez, R. Industrial biomanufacturing: the future of chemical production. Science 355, aag0804 (2017).

7. Schirmer, A., Rude, M. A., Li, X., Popova, E. \& del Cardayre, S. B. Microbial biosynthesis of alkanes. Science 329, 559-562 (2010)

8. Rude, M. A. et al. Terminal olefin (1-alkene) biosynthesis by a novel P450 fatty acid decarboxylase from Jeotgalicoccus species. Appl. Environ. Microbiol. 77, 1718-1727 (2011).

9. Rui, Z. et al. Microbial biosynthesis of medium-chain 1-alkenes by a nonheme iron oxidase. Proc. Natl Acad. Sci. 111, 18237-18242 (2014).

10. Rui, Z., Harris, N. C., Zhu, X., Huang, W. \& Zhang, W. Discovery of a family of desaturase-like enzymes for 1-alkene biosynthesis. ACS Catal. 5, 7091-7094 (2015).

11. Sorigué, D. et al. An algal photoenzyme converts fatty acids to hydrocarbons. Science 357, 903-907 (2017).

12. Blazeck, J. et al. Harnessing Yarrowia lipolytica lipogenesis to create a platform for lipid and biofuel production. Nat. Commun. 5, 3131 (2014).

13. Xu, P., Qiao, K., Ahn, W. S. \& Stephanopoulos, G. Engineering Yarrowia lipolytica as a platform for synthesis of drop-in transportation fuels and oleochemicals. Proc. Natl Acad. Sci. U. S. A. 113, 10848-10853 (2016).

14. Bruder, S., Moldenhauer, E. J., Lemke, R. D., Ledesma-Amaro, R. \& Kabisch, J. Drop-in biofuel production using fatty acid photodecarboxylase from Chlorella variabilis in the oleaginous yeast Yarrowia lipolytica. Biotechnol. Biofuels 12, 1-13 (2019).

15. Huijbers, M. M. E., Zhang, W., Tonin, F. \& Hollmann, F. Light-driven enzymatic decarboxylation of fatty acids. Angew. Chem. Int. Ed. 57, 13648-13651 (2018).

16. Yuzbasheva, E. Y. et al. A metabolic engineering strategy for producing free fatty acids by the Yarrowia lipolytica yeast based on impairment of glycerol metabolism. Biotechnol. Bioeng. 115, 433-443 (2018).

17. Yunus, I. S. et al. Synthetic metabolic pathways for photobiological conversion of $\mathrm{CO}_{2}$ into hydrocarbon fuel. Metab. Eng. 49, 201-211 (2018).

18. Rutter, C. D., Zhang, S. \& Rao, C. V. Engineering Yarrowia lipolytica for production of medium-chain fatty acids. Appl. Microbiol. Biotechnol. 99, 7359-7368 (2015).

19. Tenagy et al. Involvement of acyl-CoA synthetase genes in n-alkane assimilation and fatty acid utilization in yeast Yarrowia lipolytica. FEMS Yeast Res. 15, 1-12 (2015)

20. Ledesma-Amaro, R., Dulermo, R., Niehus, X. \& Nicaud, J. M. Combining metabolic engineering and process optimization to improve production and secretion of fatty acids. Metab. Eng. 38, 38-46 (2016).
21. Cantu, D. C., Chen, Y. \& Reilly, P. J. Thioesterases: a new perspective based on their primary and tertiary structures. Protein Sci. 19, 1281-1295 (2010).

22. Kotaka, M. et al. Structure and catalytic mechanism of the thioesterase CalE7 in enediyne biosynthesis. J. Biol. Chem. 284, 15739-15749 (2009).

23. Eser, B. E., Zhang, X., Chanani, P. K., Begley, T. P. \& Ealick, S. E. From suicide enzyme to catalyst: the iron-dependent sulfide transfer in Methanococcus jannaschii thiamin thiazole biosynthesis. J. Am. Chem. Soc. 138, 3639-3642 (2016).

24. Tai, M. \& Stephanopoulos, G. Engineering the push and pull of lipid biosynthesis in oleaginous yeast Yarrowia lipolytica for biofuel production. Metab. Eng. 15, 1-9 (2013).

25. Zhang, H., Damude, H. G. \& Yadav, N. S. Three diacylglycerol acyltransferases contribute to oil biosynthesis and normal growth in Yarrowia lipolytica. Yeast 29, 25-38 (2012).

26. Jiménez-Díaz, L., Caballero, A., Pérez-Hernández, N. \& Segura, A. Microbial alkane production for jet fuel industry: motivation, state of the art and perspectives. Microb. Biotechnol. 10, 103-124 (2017).

27. Peralta-Yahya, P. P., Zhang, F., del Cardayre, S. B. \& Keasling, J. D. Microbial engineering for the production of advanced biofuels. Nature 488, 320-328 (2012).

28. Park, J. O. et al. Synergistic substrate cofeeding stimulates reductive metabolism. Nat. Metab. 1, 643-651 (2019).

29. Palmqvist, E. \& Hahn-Hägerdal, B. Fermentation of lignocellulosic hydrolysates. II: inhibitors and mechanisms of inhibition. Bioresour. Technol 74, 25-33 (2000).

30. Choi, Y. J. \& Lee, S. Y. Microbial production of short-chain alkanes. Nature 502, 571-574 (2013).

31. Dennig, A. et al. Oxidative decarboxylation of short-chain fatty acids to 1 alkenes. Angew. Chem. Int. Ed. 54, 8819-8822 (2015).

32. Herman, N. A. \& Zhang, W. Enzymes for fatty acid-based hydrocarbon biosynthesis. Curr. Opin. Chem. Biol. 35, 22-28 (2016).

33. Liu, N., Qiao, K. \& Stephanopoulos, G. 13C metabolic flux analysis of acetate conversion to lipids by Yarrowia lipolytica. Metab. Eng. 38, 86-97 (2016).

34. Beopoulos, A. et al. Yarrowia lipolytica as a model for bio-oil production. Prog. Lipid Res. 48, 375-387 (2009).

35. Maleki, N. \& Eiteman, M. A. Recent progress in the microbial production of pyruvic acid. Fermentation 3, 1-17 (2017).

36. Max, B. et al. Biotechnological production of citric acid. Braz. J. Microbiol. 41, 862-875 (2010).

37. Qiao, K., Wasylenko, T. M., Zhou, K., Xu, P. \& Stephanopoulos, G. Lipid production in Yarrowia lipolytica is maximized by engineering cytosolic redox metabolism. Nat. Biotechnol. 35, 173-177 (2017).

38. Guo, H., Madzak, C., Du, G., Zhou, J. \& Chen, J. Effects of pyruvate dehydrogenase subunits overexpression on the $\alpha$-ketoglutarate production in Yarrowia lipolytica WSH-Z06. Appl. Microbiol. Biotechnol. 98, 7003-7012 (2014).

39. Liu, H., Marsafari, M., Wang, F., Deng, L. \& Xu, P. Engineering acetyl-CoA metabolic shortcut for eco-friendly production of polyketides triacetic acid lactone in Yarrowia lipolytica. Metab. Eng. 56, 60-68 (2019).

40. Zhou, J., Yin, X., Madzak, C., Du, G. \& Chen, J. Enhanced a-ketoglutarate production in Yarrowia lipolytica WSH-Z06 by alteration of the acetyl-CoA metabolism. J. Biotechnol. 161, 257-264 (2012).

41. Ledesma-Amaro, R. et al. Metabolic engineering of Yarrowia lipolytica to produce chemicals and fuels from xylose. Metab. Eng. 38, 115-124 (2016).

42. McIntosh, S. \& Vancov, T. Optimisation of dilute alkaline pretreatment for enzymatic saccharification of wheat straw. Biomass-. Bioenergy 35, 3094-3103 (2011)

43. Schwartz, C. M., Hussain, M. S., Blenner, M. \& Wheeldon, I. Synthetic RNA polymerase III promoters facilitate high-efficiency CRISPR-Cas9-mediated genome editing in Yarrowia lipolytica. ACS Synth. Biol. 5, 356-359 (2016).

44. Pettersen, E. F. et al. UCSF Chimera-A visualization system for exploratory research and analysis. J. Comput. Chem. 25, 1605-1612 (2004).

45. Trott, O. \& Olson, A. J. AutoDock Vina: Improving the speed and accuracy of docking with a new scoring function, efficient optimization, and multithreading. J. Comput. Chem. 31, 455-461 (2009).

46. Takai, H. et al. Construction and characterization of a Yarrowia lipolytica mutant lacking genes encoding cytochromes P450 subfamily 52. Fungal Genet. Biol. 49, 58-64 (2012).

\section{Acknowledgements}

The authors thank the technical assistance from Dr. Yiming Mo, Eric Ryan Powers, and Seungkyun Ha at Department of Chemical Engineering, Massachusetts Institute of Technology for measuring spectra and LED power of the blue light bulbs. We thank Yuexuan $\mathrm{Zu}$ and Dr. Irum Perveen at Department of Chemical Engineering, Massachusetts Institute of Technology for providing pET28 vector backbone and reagents for protein purification. This work was supported by the Independent Research Fund Denmark, International Postdoc Grant (7026-00039B). 


\section{Author contributions}

J.L., Z.G., P.R.J., and G.S. initiated the project. J.L., Y.M., N.L., and G.S. designed the experiments. J.L. performed experiments, analyzed data, and drafted the manuscript. B.E. E. performed molecular docking and wrote decarboxylation mechanism. All authors contributed to discussions of the data and to the writing of the manuscript.

\section{Competing interests}

The authors declare no competing interests.

\section{Additional information}

Supplementary information is available for this paper at https://doi.org/10.1038/s41467020-19995-0.

Correspondence and requests for materials should be addressed to G.S.

Peer review information Nature Communications thanks the anonymous reviewers for their contribution to the peer review of this work.
Reprints and permission information is available at http://www.nature.com/reprints

Publisher's note Springer Nature remains neutral with regard to jurisdictional claims in published maps and institutional affiliations.

\section{(c) (i)}

Open Access This article is licensed under a Creative Commons Attribution 4.0 International License, which permits use, sharing, adaptation, distribution and reproduction in any medium or format, as long as you give appropriate credit to the original author(s) and the source, provide a link to the Creative Commons license, and indicate if changes were made. The images or other third party material in this article are included in the article's Creative Commons license, unless indicated otherwise in a credit line to the material. If material is not included in the article's Creative Commons license and your intended use is not permitted by statutory regulation or exceeds the permitted use, you will need to obtain permission directly from the copyright holder. To view a copy of this license, visit http://creativecommons.org/licenses/by/4.0/.

(C) The Author(s) 2020 